\title{
Boundary concentration phenomena for the higher-dimensional Keller-Segel system
}

\author{
Oscar Agudelo • Angela Pistoia
}

Received: date / Accepted: date

\begin{abstract}
We study the existence of steady states to the Keller-Segel system with linear chemotactical sensitivity function on a smooth bounded domain in $\mathbb{R}^{N}, N \geq 3$, having rotational symmetry. We find three different types of chemoattractant concentration which concentrate along suitable $(N-$ 2 )-dimensional minimal submanifolds of the boundary. The corresponding density of the cellular slime molds exhibit in the limit one or more Dirac measures supported on those boundary submanifolds.
\end{abstract}

Keywords Keller-Segel system · boundary and interior concentration layer

Mathematics Subject Classification (2000) 35J60 (primary) - 35B33 . 35J20 (secondary)

\section{Contents}

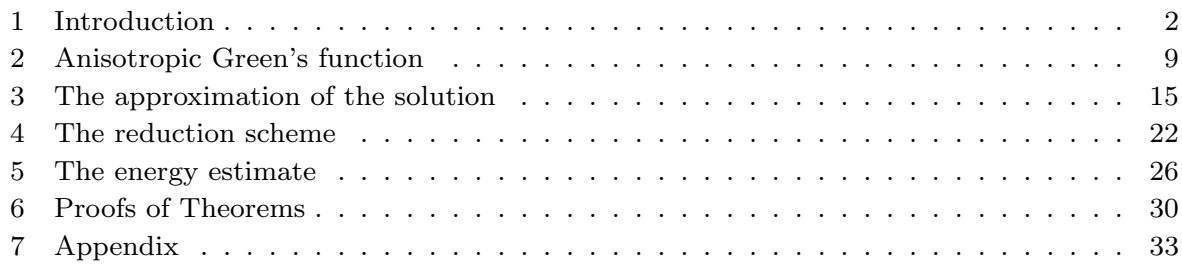

Oscar Agudelo

NTIS Department of Mathematics, Západočeská Univerzita v Plzni

E-mail: oiagudel@ntis.zcu.cz

Angela Pistoia

Dipartimento di Scienze Base e Applicate La Sapienza Universitá di Roma

E-mail: angela.pistoia@uniroma1.it 


\section{Introduction}

In 1970 Keller and Segel 23 presented a system of two strongly coupled parabolic PDE's to describe the aggregation of cellular slime molds like Dictyostelium Discoidem. Assuming the whole process to take place on a suitable bounded region $D$ in $\mathbb{R}^{N}, N \geq 1$, with no flux across the boundary, the myxoamoebae density of the cellular slime molds $w(t, x)$ and the chemoattractant concentration $v(t, x)$ at time $t$ in a point $x$ in $D$ satisfy the system

$$
\left\{\begin{array}{l}
\partial_{t} w=\nabla[\mu(w, v) \nabla w-\chi(w, v) \nabla v] \text { in } \mathbb{R} \times D \\
\partial_{t} v=\gamma_{0} \Delta v+k(w, v) \text { in } \mathbb{R} \times D \\
\partial_{\nu} w=\partial_{\nu} v=0 \text { in } \mathbb{R} \times \partial D \\
w(0, x)=w_{0}(x), v(0, x)=v_{0}(x) \text { in } D,
\end{array}\right.
$$

where

$-\mu(w, v)$ is the random motility coefficient

- $\chi(w, v)=\chi_{0} \mu(w, v) w \nabla \Phi(v)$ is the total chemotaxic flux, where $\chi_{0}$ is a constant and $\Phi$ is a smooth increasing function called chemotactic sensitivity function

$-\gamma_{0}>0$ is a constant diffusion coefficient

$-k(w, v)$ models the reaction, which commonly is $k(w, v)=\gamma_{0}(\alpha w-\beta v)$ for some positive constants $\alpha$ and $\beta$.

$-\nu$ is the unit inner normal derivative at the boundary.

This model has attracted the attention of many mathematicians, since it has led to a variety of stimulating problems. Many contributions have been made towards understanding analytical aspects of system (1.1). We refer the reader to $3,4,6,8,13,15,18,22$ and references therein. In particular, we quote the recent survey $[2]$ which focuses on the original model and some of its developments and is devoted to the qualitative analysis of analytic problems, such as the existence of solutions, blow-up and asymptotic behavior.

The understanding of the global dynamics of the system is strongly related to the existence of steady states, namely solutions to the system

$$
\left\{\begin{array}{l}
\nabla[\mu(w, v) \nabla w-\chi(w, v) \nabla v]=0 \text { in } D \\
\gamma_{0} \Delta v+k(w, v)=0 \text { in } D \\
\partial_{\nu} w=\partial_{\nu} v=0 \text { in } \partial D
\end{array}\right.
$$

The present paper deals with the existence of stationary solutions to the problem (1.1) when the chemotactical sensitivity function $\Phi$ is linear, i.e. $\Phi(v)=v$. In such a case the study of 1.2 reduces to a single equation. Indeed, the function $w(x)=\lambda e^{\chi_{0} v}$ solves the first equation and the second equation reduces to

$$
\Delta v+\alpha \lambda e^{\chi_{0} v}-\beta v=0 \text { in } D, \quad \partial_{\nu} v=0 \text { on } \partial D .
$$


Here we study problem 1.3 when $\lambda>0$ is small. After replacing $v(x)$ by $\chi_{0} v(x / \sqrt{\beta})$ and setting $\varepsilon^{2}=\chi_{0} \alpha \beta^{-1} \lambda$, without any loss of generality, problem (1.3) can be rewritten as

$$
\Delta v+\varepsilon^{2} e^{v}-v=0 \text { in } D, \quad \partial_{\nu} v=0 \text { on } \partial D .
$$

where $D$ is a bounded domain in $\mathbb{R}^{N}, N \geq 1, \nu$ is the inner unit normal vector to $\partial \Omega$ and $\varepsilon$ is a small parameter.

The one dimensional version of equation (1.4), was first treated by Schaaf in 27. In the two-dimensional case, Wang and Wei [30] and Senba and Suzuki 28 proved the existence of a non-costant solution for $\varepsilon \leq \varepsilon_{0}$ for some $\varepsilon_{0}$ and $\varepsilon$ possibly different from certain values depending on the domain. Successively, del Pino and Wei in [1] constructed solutions to (1.4) which concentrate (as $\varepsilon$ goes to zero) at $\kappa$ different points $\xi_{1}, \ldots, \xi_{\kappa}$ on the boundary of $D$ and $\ell$ different points $\xi_{\kappa+1}, \ldots, \xi_{\kappa+\ell}$ inside the domain $D$. In particular far away from those points the leading behavior of $v_{\varepsilon}$ is given by

$$
v_{\varepsilon}(x) \rightarrow \sum_{i=1}^{\kappa} \frac{1}{2} G\left(x, \xi_{i}\right)+\sum_{i=1}^{\ell} G\left(x, \xi_{i}\right)
$$

where $G(\cdot, \xi)$ is the Green function for the problem

$$
\left\{\begin{array}{l}
-\Delta G+G=8 \pi \delta_{\xi}, \text { in } D, \\
\frac{\partial G}{\partial \nu}=0 \text { on } \partial D .
\end{array}\right.
$$

Here $\delta_{\zeta}$ represents the Dirac's mass concentrated at the point $\zeta$. The corresponding solution $w_{\varepsilon}(x)=\varepsilon^{2} e^{v_{\varepsilon}}$ of the first equation in 1.2 exhibits, in the limit, $\kappa$ Dirac measures on the boundary of the domain and $\ell$ Dirac measures inside the domain with respectively weights $4 \pi$ and $8 \pi$, namely

$$
w_{\varepsilon} \rightarrow \sum_{i=1}^{\kappa} 4 \pi \delta_{\xi_{i}}+\sum_{i=1}^{\ell} 8 \pi \delta_{\xi_{\kappa+i}}
$$

Recently, Del Pino, Pistoia and Vaira in 10 built a solution to problem (1.4) which concentrate along the whole boundary. In particular, far away from the boundary the leading behavior of $v_{\varepsilon}$ is given by

$$
\frac{1}{|\ln \varepsilon|} v_{\varepsilon}(x) \rightarrow \mathcal{G}(x)
$$

where $\mathcal{G}$ is the unique solution of the problem

$$
-\Delta \mathcal{G}+\mathcal{G}=0 \text { in } D, \quad \mathcal{G}=1 \text { on } \partial D .
$$

The corresponding solution $w_{\varepsilon}=\varepsilon^{2} e^{v_{\varepsilon}}$ of the first equation in 1.2 exhibits in the limit a Dirac measure supported on the boundary with a suitable weight, namely

$$
\frac{1}{|\ln \varepsilon|} w_{\varepsilon} \rightarrow-\partial_{\nu} \mathcal{G} \delta_{\partial D}
$$


$\left(\partial_{\nu} \mathcal{G}<0\right.$, because of the maximum principle and Hopf's Lemma) .

As far as we know the only results dealing with higher-dimensional cases concern the case when $D$ is a ball. Biler in 3 established the existence of strictly decreasing radial solutions, while Pistoia and Vaira in 26 found a second radial solution which is increasing and concentrates along the whole boundary of the domain as $\varepsilon$ approaches zero.

Clearly, a natural question arises.

Do there exist solutions to problem (1.4) in higher dimensional domains? In particular, is it possible to find solutions to problem (1.4) which concentrate on suitable submanifolds of the boundary as the parameter $\varepsilon$ approaches zero?

In the present paper we give a positive answer when the domain has a rotational symmetry. Let $n \in\{1,2\}$ be fixed. Let $\Omega$ be a smooth open bounded domain in $\mathbb{R}^{2}$ such that

$$
\bar{\Omega} \subset\left\{\left(x_{1}, x_{2}\right) \in \mathbb{R}^{n} \times \mathbb{R}^{2-n}: x_{i}>0, i=1, n\right\} .
$$

Let $M=\sum_{i=1}^{n} M_{i}$, where $M_{i} \geq 2$ are positive integers. Set

$$
D:=\left\{\left(y_{1}, y_{n}, x^{\prime}\right) \in \mathbb{R}^{M_{1}} \times \mathbb{R}^{M_{n}} \times \mathbb{R}^{2-n}:\left(\left|y_{1}\right|,\left|y_{n}\right|, x^{\prime}\right) \in \Omega\right\} .
$$

Then $D$ is a smooth bounded domain in $\mathbb{R}^{N}, N:=M+2-n$

The solutions we are looking for are $\Gamma$-invariant for the action of the group $\Gamma:=\mathcal{O}\left(M_{1}\right) \times \mathcal{O}\left(M_{n}\right)$ on $\mathbb{R}^{N}$ given by

$$
\left(g_{1}, g_{n}\right)\left(y_{1}, y_{n}, x^{\prime}\right):=\left(g_{1} y_{1}, g_{n} y_{n}, x^{\prime}\right) .
$$

Here $\mathcal{O}\left(M_{i}\right)$ denotes the group of linear isometries of $\mathbb{R}^{M_{i}}$.

A simple calculation shows that a function $v$ of the form

$$
v\left(y_{1}, y_{n}, x^{\prime}\right)=u\left(\left|y_{1}\right|,\left|y_{n}\right|, x^{\prime}\right)
$$

solves problem (1.4) if and only if $u$ solves the problem

$$
-\Delta u+\sum_{i=1}^{n} \frac{M_{i}-1}{x_{i}} \frac{\partial u}{\partial x_{i}}+u=\varepsilon^{2} e^{u} \quad \text { in } \Omega, \quad \partial_{\nu} u=0 \quad \text { on } \partial \Omega,
$$

which can be rewritten as

$$
-\operatorname{div}(a(x) \nabla u)+a(x) u=\varepsilon^{2} a(x) e^{u} \quad \text { in } \Omega, \quad u=0 \quad \text { on } \partial \Omega,
$$

where

$$
a\left(x_{1}, x_{n}\right):=x_{1}^{M_{1}-1} \cdot x_{n}^{M_{n}-1} .
$$

Thus, we are led to study the more general anisotropic equation

$$
-\operatorname{div}(a(x) \nabla u)+a(x) u=\varepsilon^{2} a(x) e^{u} \quad \text { in } \quad \Omega, \quad \frac{\partial u}{\partial \nu}=0 \quad \text { on } \quad \partial \Omega,
$$


where $\Omega \subset \mathbb{R}^{2}$ is a smooth bounded domain, $a: \bar{\Omega} \rightarrow \mathbb{R}$ is a strictly positive smooth function and $\varepsilon>0$ is a small parameter. Here $\nu$ stands for the inner unit normal vector to $\partial \Omega$.

Our goal is to construct solutions to problem 1.11 which concentrate at points $\zeta_{1}, \ldots, \zeta_{m}$ of the boundary of $\Omega$ as $\epsilon$ goes to 0 . They correspond via (1.9) to $\Gamma$-invariant solutions to problem 1.4 with layers which concentrate along the $\Gamma$-orbit $\Xi\left(\zeta_{i}\right)$ of $\zeta_{i}$, for $i=1, \ldots, m$ as $\epsilon$ approaches zero. Here

$$
\Xi\left(\zeta_{i}\right):=\left\{\left(y_{1}, y_{n}, x^{\prime}\right) \in \partial D:\left(\left|y_{1}\right|,\left|y_{n}\right|, x^{\prime}\right)=\zeta_{i} \in \partial \Omega\right\}
$$

is a $(N-2)$-dimensional minimal submanifold of the boundary of $D$ diffeomorphic to $\mathbb{S}^{M_{1}-1} \times \mathbb{S}^{M_{n}-1}$ where $\mathbb{S}^{M_{i}-1}$ is the unit sphere in $\mathbb{R}^{M_{i}}$.

In order to state our main result, we need to introduce some tools.

The basic cell in our construction are the so-called standard bubbles

$$
U_{\mu, \zeta}(x):=\ln \left(\frac{8 \mu^{2}}{\left(\mu^{2}+|x-\zeta|^{2}\right)^{2}}\right), x, \zeta \in \mathbb{R}^{2}, \mu>0,
$$

which solve the Liouville equation

$$
\Delta U+e^{U}=0 \text { in } \mathbb{R}^{2} .
$$

To get a good approximation, we need to project the bubbles in order to fit the Neumann boundary condition with the linear differential operator

$$
\mathcal{L}_{a} u:=\Delta u+\nabla(\ln a) \cdot \nabla u-u,
$$

namely

$$
\mathcal{L}_{a} P U_{\mu, \zeta}=\mathcal{L}_{a} U_{\mu, \zeta} \text { in } \Omega, \partial_{\nu} P U_{\mu, \zeta}=0 \text { on } \partial \Omega .
$$

To compute the error given by the projected bubble, we need to perform a careful analysis of the regularity and asymptotic behavior of the Green's function $G_{a}(\cdot, \zeta)$ associate with $\mathcal{L}_{a}$ with Neumann boundary condition, namely

$$
\left\{\begin{array}{l}
\mathcal{L}_{a} G_{a}(\cdot, \zeta)+8 \pi \delta_{\zeta}=0 \quad \text { in } \quad \Omega \\
\frac{\partial G_{a}(\cdot, \zeta)}{\partial \nu}=0 \quad \text { on } \quad \partial \Omega
\end{array}\right.
$$

for every $\zeta \in \bar{\Omega}$. The regular part of $G_{a}(\cdot, \zeta)$ is defined for $x \in \Omega$ as

$$
H_{a}(x, \zeta):= \begin{cases}G_{a}(x, \zeta)+4 \ln (|x-\zeta|), & \zeta \in \Omega, \\ G_{a}(x, \zeta)+8 \ln (|x-\zeta|), & \zeta \in \partial \Omega .\end{cases}
$$

Now, we can state our main results.

Our first result concerns with the existence of solutions whose concentration points are inside the domain and approach different points of the boundary as $\varepsilon$ goes to zero. 
Theorem 1 Assume

(A1) there exist $m$ different points $\zeta_{1}^{*}, \ldots, \zeta_{m}^{*} \in \partial \Omega$ such that $\zeta_{i}^{*}$ is either a strict local maximum or a strict local minimum point of a restricted to $\partial \Omega$, satisfying that

$$
\partial_{\nu} a\left(\zeta_{i}^{*}\right):=\nabla a\left(\zeta_{i}^{*}\right) \cdot \nu\left(\zeta_{i}^{*}\right)<0, \quad \forall i=, \ldots, m .
$$

Then if $\varepsilon>0$ is small enough there exist $m$ points $\zeta_{1}^{\varepsilon}, \ldots, \zeta_{m}^{\varepsilon} \in \Omega, m$ positive real numbers $d_{1}^{\varepsilon}, \ldots, d_{m}^{\varepsilon}$ and a positive solution $u_{\varepsilon}$ of equation (1.11) such that

$$
u_{\varepsilon}(x):=\sum_{i=1}^{m} \ln \left(\frac{1}{\left(d_{i}^{\varepsilon}+\left|x-\zeta_{i}^{\varepsilon}\right|^{2}\right)^{2}}\right)+H_{a}\left(x, \zeta_{i}^{\varepsilon}\right)+o(1), \quad \forall x \in \bar{\Omega},
$$

where as $\varepsilon \rightarrow 0$

$$
\zeta_{i}^{\varepsilon} \rightarrow \zeta_{i}^{*}, \quad \operatorname{dist}\left(\zeta_{i}^{\varepsilon}, \partial \Omega\right)=\mathcal{O}\left(|\ln (\varepsilon)|^{-1}\right)
$$

and

$$
c \ln \left(\frac{1}{\varepsilon}\right) \leq d_{i}^{\varepsilon} \leq C \ln \left(\frac{1}{\varepsilon}\right) .
$$

for some positive constants $c$ and $C$.

In particular,

$$
u_{\varepsilon}(x) \rightarrow \sum_{i=1}^{m} G_{a}\left(x, \zeta_{i}^{*}\right) \text { in } \Omega \backslash \cup_{i=1}^{m} B\left(\zeta_{i}^{*}, r\right) \text { as } \varepsilon \rightarrow 0
$$

for some $r>0$ and

$$
\varepsilon^{2} \int_{\Omega} e^{u_{\varepsilon}(x)} d x \rightarrow 8 \pi m \text { as } \varepsilon \rightarrow 0 .
$$

Our second result concerns with the existence of solutions whose concentration points lie on the boundary and are far away from each other as $\varepsilon$ goes to zero.

Theorem 2 Assume

(A2) there exist $m$ different points $\zeta_{1}^{*}, \ldots, \zeta_{m}^{*} \in \partial \Omega$ such that $\zeta_{i}^{*}$ is either a strict local maximum or a strict local minimum point of a restricted to $\partial \Omega$.

Then if $\varepsilon>0$ is small enough there exist $m$ points $\zeta_{1}^{\varepsilon}, \ldots, \zeta_{m}^{\varepsilon} \in \partial \Omega, m$ positive real numbers $d_{1}^{\varepsilon}, \ldots, d_{m}^{\varepsilon}$ and a positive solution $u_{\varepsilon}$ of equation (1.11) such that

$$
u_{\varepsilon}(x):=\sum_{i=1}^{m} \ln \left(\frac{1}{\left(\left|d_{i}^{\varepsilon}\right|^{2}+\left|x-\zeta_{i}^{\varepsilon}\right|^{2}\right)^{2}}\right)+\frac{1}{2} H_{a}\left(x, \zeta_{i}^{\varepsilon}\right)+o(1), \quad \forall x \in \bar{\Omega}
$$

where as $\varepsilon \rightarrow 0$,

$$
\zeta_{i}^{\varepsilon} \rightarrow \zeta_{i}^{*} \text { as } \varepsilon \rightarrow 0 \quad \text { and } \quad c \leq d_{i}^{\varepsilon} \leq C,
$$


for some positive constants $c$ and $C$.

In particular,

$$
u_{\varepsilon}(x) \rightarrow \sum_{i=1}^{m} \frac{1}{2} G_{a}\left(x, \zeta_{i}^{*}\right) \text { in } \Omega \backslash \cup_{i=1}^{m} B\left(\zeta_{i}^{*}, r\right) \text { as } \varepsilon \rightarrow 0
$$

for some $r>0$ and

$$
\varepsilon^{2} \int_{\Omega} e^{u_{\varepsilon}(x)} d x \rightarrow 4 \pi m \text { as } \varepsilon \rightarrow 0
$$

Our last existence result concerns with the existence of solutions whose concentration points lie on the boundary and collapse to the same point as $\varepsilon$ goes to zero.

\section{Theorem 3 Assume}

(A3) $\zeta_{0} \in \partial \Omega$ is a strict local maximum point of a restricted to $\partial \Omega$.

Then for any integer $m \geq 1$ if $\varepsilon>0$ small enough, there exist $m$ points $\zeta_{1}^{\varepsilon}, \ldots, \zeta_{m}^{\varepsilon} \in \partial \Omega$, and positive real numbers $d_{1}^{\varepsilon}, \ldots, d_{m}^{\varepsilon}$ and a positive solution $u_{\varepsilon}$ of equation (1.11) such that

$$
u_{\varepsilon}(x):=\sum_{i=1}^{m} \ln \left(\frac{1}{\left(\left|d_{i}^{\varepsilon}\right|^{2}+\left|x-\zeta_{i}^{\varepsilon}\right|^{2}\right)^{2}}\right)+\frac{1}{2} H_{a}\left(x, \zeta_{i}^{\varepsilon}\right)+o(1), \quad \forall x \in \bar{\Omega},
$$

where as $\varepsilon \rightarrow 0$,

$$
\zeta_{i}^{\varepsilon} \rightarrow \zeta_{0} \quad \text { and } \quad c \ln \left(\frac{1}{\varepsilon}\right) \leq d_{i}^{\varepsilon} \leq C \ln \left(\frac{1}{\varepsilon}\right),
$$

for some positive constants $c$ and $C$.

In particular,

$$
u_{\varepsilon}(x) \rightarrow \frac{m}{2} G_{a}\left(x, \zeta_{0}\right) \text { in } \Omega \backslash B\left(\zeta_{0}, r\right) \text { as } \varepsilon \rightarrow 0
$$

for some $r>0$ and

$$
\varepsilon^{2} \int_{\Omega} e^{u_{\varepsilon}(x)} d x \rightarrow 4 \pi m \text { as } \varepsilon \rightarrow 0
$$

All the previous arguments yield immediately the following existence result for the higher-dimensional problem (1.4).

Theorem 4 Assume $D$ is as described in 1.8 . 
(i) If (A1) of Theorem 1 holds, then for every $\varepsilon>0$ small enough, there exists a $\Gamma$-invariant solution $v_{\varepsilon}$ to problem (1.4) with $m$ interior layers which concentrate at $m$ distinct $(N-2)$-dimensional minimal submanifold of the boundary of $D$ as $\varepsilon \rightarrow 0$. Moreover (see 1.12)

$$
\varepsilon^{2} \int_{\mathcal{D}} e^{v_{\varepsilon}(x)} d x \rightarrow 8 \pi \sum_{i=1}^{m}\left|\Xi\left(\zeta_{i}^{*}\right)\right| \text { as } \varepsilon \rightarrow 0 .
$$

(ii) If (A2) of Theorem 2 holds, then for every $\varepsilon>0$ small enough, there exists a $\Gamma$-invariant solution $v_{\varepsilon}$ to problem (1.4) with $m$ boundary layers which concentrate at $m$ distinct $(N-2)$-dimensional minimal submanifold of the boundary of $D$ as $\varepsilon \rightarrow 0$. Moreover (see (1.12)

$$
\varepsilon^{2} \int_{\mathcal{D}} e^{v_{\varepsilon}(x)} d x \rightarrow 4 \pi \sum_{i=1}^{m}\left|\Xi\left(\zeta_{i}^{*}\right)\right| \text { as } \varepsilon \rightarrow 0 .
$$

(iii) If (A3) of Theorem 3, then for any integer $m \geq 1$ and every $\varepsilon>0$ small enough, there exists a $\Gamma$-invariant solution $v_{\varepsilon}$ to problem $(1.4)$ with $m$ boundary layers which concentrate at the same $(N-2)$-dimensional minimal submanifold of the boundary of $D$ as $\varepsilon \rightarrow 0$. Moreover (see (1.12)

$$
\varepsilon^{2} \int_{\mathcal{D}} e^{v_{\varepsilon}(x)} d x \rightarrow 4 \pi m\left|\Xi\left(\zeta_{0}\right)\right| \text { as } \varepsilon \rightarrow 0 .
$$

Let us make some comments.

First, the symmetry assumption allows to simplify in a considerable way the computations, but the phenomena we get should be present in a more general domain. We strongly believe that our results hold true even if we drop the symmetry assumption. In particular, we conjecture that (i) and (ii) of Theorem 4 can be rephrased in the more general form

(1) if $D$ is a general bounded domain in $\mathbb{R}^{N}$ with $N \geq 3$ and $\Xi$ is a $(N-$ 2)-dimensional minimal submanifold (possibly non-degenerate) of the boundary of $D$ with a suitable sign on the sectional curvatures, then problem (1.4) has a solution with an interior layer concentrating along $\Xi$ as $\varepsilon$ goes to zero.

(2) if $D$ is a general bounded domain in $\mathbb{R}^{N}$ with $N \geq 3$ and $\Xi$ is a $(N-$ 2)-dimensional minimal submanifold (possibly non-degenerate) of the boundary of D, then problem (1.4) has a solution with a boundary layer concentrating along $\Xi$ as $\varepsilon$ goes to zero. 
Second, the proof of our result relies on a well known Lyapunov-Schmidt procedure. The same strategy has been used by Wei, Ye and Zhou 32, 33 to find concentrating solutions for the anisotropic Dirichlet problem

$$
-\operatorname{div}(a(x) \nabla u)=\varepsilon^{2} a(x) e^{u} \quad \text { in } \quad \Omega, \quad u=0 \quad \text { on } \quad \partial \Omega,
$$

where $\Omega \subset \mathbb{R}^{2}$ is a smooth bounded domain, $a: \bar{\Omega} \rightarrow \mathbb{R}$ is a strictly positive smooth function and $\varepsilon>0$ is a small parameter.

The structure of the paper is the following. In section 2 we perform a careful study of Green's function introduced in 1.16. In section 3 we provide the approximation of the solutions predicted by our existence theorems and compute the error created by this approximation. Section 4 concerns with the finite dimensional reduction scheme which is the first step in the proof of our existence results. In section 5 we find precise energy estimates for the approximation found in section 3. Finally, in section 6 we provide the detailed proof of our existence theorems using variational and topological arguments.

\section{Anisotropic Green's function}

In this part we analyze the asymptotic boundary behavior of the functions $G(\cdot, \zeta)=G_{a}(\cdot, \zeta)$ and $H(\cdot, \zeta)=H_{a}(\cdot, \zeta)$ introduced in (1.16) and 1.17), respectively.

We begin by recalling some well known facts about Sobolev spaces and refer the reader to [5, 12, 16] and references therein, for an exhaustive description of these spaces and the related results hereby presented.

Let $\Omega \subset \mathbb{R}^{2}$ be a bounded domain with smooth boundary. The space $L^{p}(\Omega)$ is the space of measurable functions $v: \Omega \rightarrow \mathbb{R}$ for which the norm

$$
\|v\|_{L^{p}(\Omega)}:=\left\{\begin{array}{c}
\left(\int_{\Omega}|v(x)|^{p} d x\right)^{\frac{1}{p}}, 1 \leq p<\infty \\
\sup _{x \in \Omega}|v(x)|, \quad p=\infty
\end{array}\right.
$$

is finite. The Sobolev space $W^{k, p}(\Omega)$ is the space of functions in $L^{p}(\Omega)$ having weak derivatives, up to order $k$, also in $L^{p}(\Omega)$. The space $W^{k, p}(\Omega)$ is a Banach space endowed with the norm

$$
\|v\|_{W^{k, p}(\Omega)}:=\sum_{i=0}^{k}\left\|D^{i} v\right\|_{L^{p}(\Omega)} .
$$

Given $k \in \mathbb{N}$, we let $C^{k}(\bar{\Omega})$ denote the space of functions having continuous derivatives of order $k$ up to the boundary. In addition, for any $\alpha \in(0,1]$, we denote $C^{k, \alpha}(\bar{\Omega})$ the Hölder space, consisting of functions $v \in C^{k}(\bar{\Omega})$ for which the Hölder norm

$$
\|v\|_{C^{k, \alpha}(\bar{\Omega})}:=\sum_{i=0}^{k}\left\|D^{i} v\right\|_{L^{\infty}(\Omega)}+\sup _{x, y \in \bar{\Omega} x \neq y} \frac{\left|D^{i} v(x)-D^{i} v(y)\right|}{|x-y|^{\alpha}}
$$


is finite.

We will make use of the following embeddings for Sobolev functions.

$$
W^{2, p}(\Omega) \hookrightarrow\left\{\begin{array}{cc}
W^{1, \frac{2 p}{2-p}}(\Omega) \cap C^{0,2\left(1-\frac{1}{p}\right)}(\bar{\Omega}), & 1<p<2 \\
C^{1,1-\frac{2}{p}}(\bar{\Omega}), & p>2 .
\end{array}\right.
$$

From the continuity of the trace operator together with Sobolev embeddings in one dimensional manifolds, we find that for any $1<p<2$,

$$
W^{1, p}(\Omega) \hookrightarrow L^{q}(\partial \Omega), \quad \forall q \in\left(1, \frac{p}{2-p}\right) .
$$

Set

$$
\gamma(x)=(\nabla \ln a)(x), \quad \text { for } x \in \bar{\Omega}
$$

and notice that $\gamma \in C^{\infty}(\bar{\Omega})$, since $a \in C^{\infty}(\bar{\Omega})$ and $a>0$.

Recall also from (1.15) that

$$
\mathcal{L}:=\Delta+\gamma(x) \cdot \nabla-1
$$

and that $G=G(x, \zeta)$, the Green's function associated to $\mathcal{L}$ satisfies for every $\zeta \in \bar{\Omega}$ the boundary value problem

$$
\begin{aligned}
\mathcal{L} G(\cdot, \zeta)+8 \pi \delta_{\zeta} & =0 \quad \text { in } \quad \Omega, \\
\frac{\partial G(\cdot, \zeta)}{\partial \nu}=0 \quad & \text { on } \quad \partial \Omega .
\end{aligned}
$$

For $x \in \Omega$, the regular part of $G(x, \zeta)$ is the function

$$
H(x, \zeta):= \begin{cases}G(x, \zeta)+4 \ln (|x-\zeta|), & \zeta \in \Omega, \\ G(x, \zeta)+8 \ln (|x-\zeta|), & \zeta \in \partial \Omega\end{cases}
$$

Let us introduce the vector function $R=R(z)$, solving

$$
\Delta_{z} R-R=\frac{z}{|z|^{2}} \quad \text { in } \mathbb{R}^{2}, \quad R \in L_{\text {loc }}^{\infty}\left(\mathbb{R}^{2}\right) .
$$

We remark that standard regularity theory implies that $R \in W_{\text {loc }}^{2, p}\left(\mathbb{R}^{2}\right) \cap$ $C^{\infty}\left(\mathbb{R}^{2}-\{0\}\right)$, for any $p \in(1,2)$. On the other hand, Sobolev embeddings allow us to conclude that for any ball $B_{r}(0)$ of radius $r>0$ and centered at the origin

$$
R \in W^{1, p}\left(B_{r}(0)\right) \cap C^{0, \frac{1}{p}}\left(\overline{B_{r}(0)}\right), \quad \forall p \in(1, \infty) .
$$

Our first result uses the function $R(z)$ to describe the regularity of the family of functions $\zeta \in \bar{\Omega} \mapsto H(\cdot, \zeta)$ and concerns with the local behavior of $H(x, \zeta)$. 
Theorem 5 Let $R=R(z)$ be the function described in (2.6). There exists a function $H_{1}=H_{1}(x, \zeta)$, such that

(i) for every $\zeta, x \in \bar{\Omega}$,

$$
H(x, \zeta)=H_{1}(x, \zeta)+\left\{\begin{array}{l}
4 \gamma(\zeta) \cdot R(x-\zeta), \zeta \in \Omega \\
8 \gamma(\zeta) \cdot R(x-\zeta), \zeta \in \partial \Omega
\end{array}\right.
$$

and

(ii) the mapping $\zeta \in \bar{\Omega} \mapsto H_{1}(\cdot, \zeta)$ belongs to $C^{1}\left(\Omega ; C^{1}(\bar{\Omega})\right) \cap C^{1}\left(\partial \Omega ; C^{1}(\bar{\Omega})\right)$.

In particular, for any $\alpha \in(0,1), H \in C^{0, \alpha}(\bar{\Omega} \times \Omega) \cap C^{0, \alpha}(\bar{\Omega} \times \partial \Omega)$ and the Robin's function $\zeta \in \bar{\Omega} \mapsto H(\zeta, \zeta)$ belongs to $C^{1}(\Omega) \cap C^{1}(\partial \Omega)$.

Proof For $\zeta \in \bar{\Omega}$, let us write

$$
c:=\left\{\begin{array}{l}
4, \text { if } \zeta \in \Omega \\
8, \text { if } \zeta \in \partial \Omega .
\end{array}\right.
$$

From 2.3 and $(2.5)$, we observe that

$$
\mathcal{L} H(x, \zeta)=c \gamma(x) \cdot \frac{x-\zeta}{|x-\zeta|^{2}}-c \ln (|x-\zeta|), \quad \forall x \in \Omega
$$

with the boundary condition

$$
\frac{\partial H(x, \zeta)}{\partial \nu_{x}}=c \nu(x) \cdot \frac{x-\zeta}{|x-\zeta|^{2}}, \quad \forall x \in \partial \Omega, \quad x \neq \zeta .
$$

The right hand side in 2.10 can be written as

$$
c \gamma(\zeta) \cdot \frac{x-\zeta}{|x-\zeta|^{2}}+E(x, \zeta)
$$

where

$$
E(x, \zeta):=c(\gamma(x)-\gamma(\zeta)) \cdot \frac{x-\zeta}{|x-\zeta|^{2}}-c \ln (|x-\zeta|) .
$$

Using a smooth extension of the function $\gamma$ to a larger compact domain containing $\Omega$, we find a constant $C>0$ depending only on $\gamma$ and $\Omega$ such that

$$
\left|(\gamma(x)-\gamma(\zeta)) \cdot \frac{(x-\zeta)}{|x-\zeta|^{2}}\right| \leq C, \quad \text { for } x \in \Omega .
$$

On the other hand, given $p \in(0,1)$, there exists a constant $C=C(p, \Omega)>$ 0 , such that for every $\zeta \in \bar{\Omega}$,

$$
\int_{\Omega}|\ln (|x-\zeta|)|^{p} d x \leq C \int_{0}^{2 \operatorname{diam}(\Omega)} r|\ln (r)|^{p} d r \leq C .
$$


We conclude that for any $p \in(1, \infty)$, the mapping

$$
\zeta \in \bar{\Omega} \mapsto E(\cdot, \zeta) \in L^{p}(\Omega)
$$

is well defined. The Dominated Convergence Theorem yields that $\zeta \mapsto E(\cdot, \zeta)$ belongs to $C\left(\Omega ; L^{p}(\Omega)\right) \cap C\left(\partial \Omega ; L^{p}(\Omega)\right)$.

Next, let $I_{2 \times 2}$ be the $2 \times 2$ identity matrix and

$$
(x-\zeta) \otimes(x-\zeta):=\left[\begin{array}{cc}
\left(x_{1}-\zeta_{1}\right)^{2} & \left(x_{1}-\zeta_{1}\right)\left(x_{2}-\zeta_{2}\right) \\
\left(x_{1}-\zeta_{1}\right)\left(x_{2}-\zeta_{2}\right) & \left(x_{2}-\zeta_{2}\right)^{2}
\end{array}\right] .
$$

We compute for $x, \zeta \in \bar{\Omega}, x \neq \zeta$

$$
\begin{gathered}
\nabla_{\zeta} E(x, \zeta)= \\
c\left(D \gamma(\zeta) \cdot \frac{x-\zeta}{|x-\zeta|^{2}}+\frac{4(\gamma(x)-\gamma(\zeta)}{|x-\zeta|^{2}} \cdot\left(I_{2 \times 2}-2 \frac{(x-\zeta) \otimes(x-\zeta)}{|x-\zeta|}\right)\right) .
\end{gathered}
$$

Using again Dominated Convergence Theorem again we obtain that for any $p \in(1,2), \zeta \in \bar{\Omega} \mapsto E(\cdot, \zeta)$ belongs to $C^{1}\left(\Omega ; L^{p}(\Omega)\right) \cap C^{1}\left(\partial \Omega ; L^{p}(\Omega)\right)$.

Define

$$
H_{1}(x, \zeta):=H(x, \zeta)-c \gamma(\zeta) \cdot R(x-\zeta), \quad \forall x, \zeta \in \Omega,
$$

where $R=R(z)$ is the vector function described in 2.6$)$ and $c$ is described in (2.9.

From 2.6), 2.10 and 2.12 , we compute the equation for $H_{1}(\cdot, \zeta)$ to obtain that

$$
\mathcal{L} H_{1}(x, \zeta)=-c \gamma(x) \cdot\left(\gamma(\zeta) \cdot D_{z} R(x-\zeta)\right)+E(x, \zeta), \quad \text { in } \Omega
$$

and the boundary condition reads as

$$
\frac{\partial H_{1}(x, \zeta)}{\partial \nu_{x}}=c \nu(x) \cdot\left(\frac{x-\zeta}{|x-\zeta|^{2}}-\gamma(\zeta) \cdot D_{z} R(x-\zeta)\right) \quad \text { on } \partial \Omega \text {. }
$$

The fact that $\zeta \mapsto E(\cdot, \zeta)$ belongs to $C\left(\Omega ; L^{p}(\Omega)\right) \cap C\left(\partial \Omega ; L^{p}(\Omega)\right)$ for any $p \in(1, \infty)$, together with $(2.7)$, imply that for any $p \in(1, \infty)$ and any $\zeta \in \bar{\Omega}$, the right hand side in equation 2.13 belongs to $L^{p}(\Omega)$.

In the case $\zeta \in \Omega$, the right hand side in 2.14 is smooth. In the case $\zeta \in \partial \Omega$, we appeal to Lemma 3 in the Appendix and embedding 2.2 to find that for any $\zeta \in \partial \Omega$ the right hand side in $\left(2.14\right.$ belongs to $L^{p}(\partial \Omega)$ for any $p>1$.

Standard elliptic regularity theory implies that for any $p \in(1, \infty), H_{1}(\cdot, \zeta) \in$ $W^{2, p}(\Omega)$ and the Sobolev embeddings in $(2.1)$ yield that $H_{1}(\cdot, \zeta) \in C^{1, \alpha}(\bar{\Omega})$, for any $\alpha \in(0,1)$. 
Finally, we check that $\zeta \mapsto H_{1}(\cdot, \zeta)$ belongs to $C^{1}\left(\Omega ; C^{1}(\bar{\Omega})\right) \cap C^{1}\left(\partial \Omega ; C^{1}(\bar{\Omega})\right)$. We first deal with the inner regularity. Recall that for any $p \in(1,2), R \in$ $W_{l o c}^{2, p}\left(\mathbb{R}^{2}\right)$ and $\zeta \mapsto \nabla_{\zeta} E$ belongs $C\left(\Omega ; L^{p}(\Omega)\right)$.

A direct application of the Dominated Convergence Theorem yields that the mapping

$$
\zeta \in \Omega \mapsto \nabla_{\zeta}\left[-c \gamma(x) \cdot\left(\gamma(\zeta) \cdot D_{z} R(x-\zeta)\right)+E(x, \zeta)\right]
$$

belongs to $C\left(\Omega ; L^{p}(\Omega)\right)$ and consequently, the mapping $\zeta \in \Omega \mapsto \nabla_{\zeta} H_{1}(\cdot, \zeta) \in$ $W^{2, p}(\Omega)$ is well defined and solves

$$
\mathcal{L}\left(\nabla_{\zeta} H_{1}(x, \zeta)\right)=\nabla_{\boldsymbol{\zeta}}\left[-c \gamma(x) \cdot\left(\gamma(\zeta) \cdot D_{z} R(x-\zeta)\right)+E(x, \zeta)\right], \quad \text { in } \Omega
$$

with the boundary condition

$$
\frac{\partial\left(\nabla_{\zeta} H_{1}(x, \zeta)\right)}{\partial \nu_{x}}=c \nabla_{\zeta}\left(\nu(x) \cdot\left(\frac{x-\zeta}{|x-\zeta|^{2}}-\gamma(\zeta) \cdot D_{z} R(x-\zeta)\right)\right) \quad \text { on } \partial \Omega \text {. }
$$

Regularity theory and Sobolev embeddings in 2.1 and 2.2 imply that the mapping $\zeta \in \Omega \mapsto \nabla_{\zeta} H_{1}(\cdot, \zeta)$ belongs to $C\left(\Omega ; C^{0, \alpha}(\Omega)\right)$ for any $\alpha \in(0,1)$.

As for the boundary regularity, we proceed in the same way as we did for the inner regularity, replacing $\nabla_{\boldsymbol{\zeta}}$ by its the tangential component respect to the $\partial \Omega$. This concludes the proof of the lemma.

Next, we introduce some notation that will be needed for subsequent developments. Fix $\eta>0$ small such that every $\zeta \in \Omega$ with $\operatorname{dist}(\zeta, \partial \Omega)<\eta$, has a well defined reflection across $\partial \Omega$ along the normal direction, $\zeta^{*} \in \Omega^{c}$. Denote,

$$
\Omega_{\eta}:=\{\zeta \in \Omega: \operatorname{dist}(\zeta, \Omega)<\eta\}
$$

which is also a smooth domain. Observe that for any $\zeta \in \Omega_{\eta},\left|\zeta-\zeta^{*}\right|=$ $2 \operatorname{dist}(\zeta, \partial \Omega)$.

Our next result concerns the boundary asymptotic behavior of the Robin's function, which we recall is given by $\zeta \in \bar{\Omega} \mapsto H(\zeta, \zeta)$.

Proposition 1 There exists a mapping $z \in C\left(\Omega_{\eta} ; C^{0, \alpha}(\bar{\Omega})\right) \cap L^{\infty}\left(\Omega_{\eta} ; C^{0, \alpha}(\bar{\Omega})\right)$ such that

$$
H(x, \zeta):=-4 \ln \left(\left|x-\zeta^{*}\right|\right)+z(x, \zeta), \quad \forall x, \in \bar{\Omega}, \quad \forall \zeta \in \Omega_{\eta} .
$$

Even more, for every $\zeta \in \Omega_{\eta}$ and $x \in \Omega$

$$
z(x, \zeta)=4 \gamma(\zeta) \cdot R(x-\zeta)-4 \gamma\left(\zeta^{*}\right) \cdot R\left(x-\zeta^{*}\right)+\tilde{z}(x, \zeta),
$$

where the mapping $\zeta \in \Omega_{\eta} \mapsto \tilde{z}(\cdot, \zeta)$ belongs to $C^{1}\left(\overline{\Omega_{\eta}} ; C^{1}(\bar{\Omega})\right)$. 
Proof Consider the function

$$
z(x, \zeta):=H(x, \zeta)+4 \ln \left(\left|x-\zeta^{*}\right|\right), \quad \forall x, \in \bar{\Omega}, \quad \forall \zeta \in \Omega_{\eta}, \quad x \neq \zeta .
$$

We directly compute from $(2.10$ and $(2.12)$, to find that

$\mathcal{L} z(\cdot, \zeta)=4 \gamma(x) \cdot\left[\frac{x-\zeta}{|x-\zeta|^{2}}-\frac{x-\zeta^{*}}{\left|x-\zeta^{*}\right|^{2}}\right]-4\left[\ln (|x-\zeta|)-\ln \left(\left|x-\zeta^{*}\right|\right)\right], \quad \forall x \in \Omega$.

with the boundary condition

$$
\frac{\partial z(x, \zeta)}{\partial \nu_{x}}=4 \nu(x) \cdot\left[\frac{x-\zeta}{|x-\zeta|^{2}}-\frac{x-\zeta^{*}}{\left|x-\zeta^{*}\right|^{2}}\right] \quad \forall x \in \partial \Omega .
$$

The right hand side in equation 2.16 can be written as

$$
4 \gamma(\zeta) \cdot \frac{x-\zeta}{|x-\zeta|^{2}}-4 \gamma\left(\zeta^{*}\right) \cdot \frac{x-\zeta^{*}}{\left|x-\zeta^{*}\right|^{2}}+\tilde{E}(x, \zeta)
$$

where

$$
\begin{aligned}
\tilde{E}(x, \zeta):=4 & (\gamma(x)-\gamma(\zeta)) \cdot \frac{x-\zeta}{|x-\zeta|^{2}}-4\left(\gamma(x)-\gamma\left(\zeta^{*}\right)\right) \cdot \frac{x-\zeta^{*}}{\left|x-\zeta^{*}\right|^{2}} \\
& -4\left[\ln (|x-\zeta|)-\ln \left(\left|x-\zeta^{*}\right|\right)\right], \quad \forall \zeta \in \Omega_{\eta}, \quad x \in \Omega .
\end{aligned}
$$

Proceeding in the same fashion as in the proof of Theorem 5 , we obtain that the mapping $\zeta \in \overline{\Omega_{\eta}} \mapsto \tilde{E}(\cdot, \zeta)$ belongs to $C^{1}\left(\overline{\Omega_{\eta}} ; L^{p}(\Omega)\right)$ for any $p \in(1, \infty)$.

To justify 2.15), we use the function $R=R(z)$, from 2.6. We decompose $z(x, \zeta)$ as

$$
z(x, \zeta)=4 \gamma(\zeta) \cdot R(x-\zeta)-4 \gamma\left(\zeta^{*}\right) \cdot R\left(x-\zeta^{*}\right)+\tilde{z}(x, \zeta)
$$

to find that if $x \in \Omega$

$$
\mathcal{L} \tilde{z}(x, \zeta)=-4 \gamma(x) \cdot\left(\gamma(\zeta) \cdot D_{z} R(x-\zeta)-\gamma\left(\zeta^{*}\right) \cdot D_{z} R\left(x-\zeta^{*}\right)\right)+\tilde{E}(x, \zeta) .
$$

with the boundary condition if $x \in \partial \Omega$

$\frac{\partial \tilde{z}(x, \zeta)}{\partial \nu_{x}}=4 \nu(x) \cdot\left[\frac{x-\zeta}{|x-\zeta|^{2}}-\frac{x-\zeta^{*}}{\left|x-\zeta^{*}\right|^{2}}-\left(\gamma(\zeta) \cdot D_{z} R(x-\zeta)-\gamma\left(\zeta^{*}\right) \cdot D_{z} R\left(x-\zeta^{*}\right)\right)\right]$.

From 2.19 and 2.20 , proceeding again as in the proof of Theorem 5 we obtain that the mapping $\zeta \in \overline{\Omega_{\eta}} \mapsto \tilde{z}(\cdot, \zeta)$ belongs to $C^{1}\left(\overline{\Omega_{\eta}} ; C^{1}(\bar{\Omega})\right)$. This concludes the proof of the proposition.

Remark: For further develoments we notice that from Proposition 1 the function $H=H(x, \zeta)$ has continuous partial derivatives in the set $\Omega \times \Omega \backslash$ $\{(x, \zeta): x \neq \zeta\}$.

Also, directly from Proposition 1 we obtain the following corollary. 
Corollary 1 Under the assumptions in Proposition 1, the Robin's function,

$$
\zeta \in \Omega \mapsto H(\zeta, \zeta)
$$

satisfies that

$$
H(\zeta, \zeta):=-4 \ln (\operatorname{dist}(\zeta, \partial \Omega))+\mathrm{z}(\zeta), \quad \forall \zeta \in \Omega_{\eta}
$$

where $\mathrm{z} \in C^{1}\left(\overline{\Omega_{\eta}}\right)$ and

$$
\mathrm{z}(\zeta):=4 \gamma(\zeta) \cdot R(0)-\gamma\left(\zeta^{*}\right) \cdot R\left(\zeta-\zeta^{*}\right)+\tilde{z}(\zeta, \zeta), \quad \forall \zeta \in \Omega_{\eta}
$$

\section{The approximation of the solution}

In this part we find an appropriate aproximation for a solution of equation 1.11 which will allow us to carry out a reduction procedure. We also compute the error created by the choice of our approximation.

To set up our approximation, we first identify the formal limit problem associated to equation (1.11).

Dividing by $a(x)$, equation 1.11 becomes

$$
\Delta u+\gamma(x) \cdot \nabla u-u+\varepsilon^{2} e^{u}=0 \quad \text { in } \Omega, \quad \frac{\partial u}{\partial \nu}=0 \quad \text { on } \partial \Omega .
$$

Take $\varepsilon>0$ and set $\Omega_{\varepsilon}:=\varepsilon^{-1} \Omega$. If $u$ is a solution of (1.11, the function

$$
v(y)=4 \ln (\varepsilon)+u(\varepsilon y), \quad y \in \Omega_{\varepsilon}
$$

solves the equation

$$
\Delta v+\varepsilon \gamma(\varepsilon y) \cdot \nabla v-\varepsilon^{2}(v-4 \ln (\varepsilon))+e^{v}=0 \quad \text { in } \Omega_{\varepsilon}, \quad \frac{\partial v}{\partial \nu_{\varepsilon}}=0 \quad \text { on } \partial \Omega_{\varepsilon}
$$

where $\nu_{\varepsilon}$ is the inner unit normal vector to $\partial \Omega_{\varepsilon}$.

From 3.3 formally as $\varepsilon \rightarrow 0^{+}$, we obtain the limit equation

$$
\Delta V+e^{V}=0, \quad \text { in } \mathbb{R}^{2}, \quad \nabla V \in L^{2}\left(\mathbb{R}^{2}\right) .
$$

Solutions to (3.4) are given by

$$
V(y):=\ln \left(\frac{8 d^{2}}{\left(d^{2}+\left|y-\zeta^{\prime}\right|^{2}\right)^{2}}\right), \quad \text { for } y \in \mathbb{R}^{2}
$$

where $d \in \mathbb{R}$ and $\zeta^{\prime} \in \mathbb{R}^{2}$ are arbitrary parameters. 
Pulling back the rescaling in 3.2 , for any $d>0$ and any $\zeta \in \mathbb{R}^{2}$, the function

$$
U_{d, \zeta}(x):=\ln \left(\frac{8 d^{2}}{\left(\varepsilon^{2} d^{2}+|x-\zeta|^{2}\right)^{2}}\right), \quad \text { for } x \in \mathbb{R}^{2}
$$

solves the equation

$$
\Delta U_{d, \zeta}+\varepsilon^{2} e^{U_{d, \zeta}}=0, \quad \text { in } \mathbb{R}^{2}, \quad \nabla_{x} U_{d, \zeta} \in L^{2}\left(\mathbb{R}^{2}\right) .
$$

Let $m \in \mathbb{N}$ be fixed. Consider $m$ real numbers $d_{i}>0$ and $m$ arbitrary different points $\zeta_{i} \in \bar{\Omega}$. For every $i=1, \ldots, m$, define

$$
U_{i}(x):=U_{d_{i}, \zeta_{i}}(x)=\ln \left(\frac{8 d_{i}^{2}}{\left(\varepsilon^{2} d_{i}^{2}+\left|x-\zeta_{i}\right|^{2}\right)^{2}}\right), \quad x \in \mathbb{R}^{2} .
$$

Let $P U_{i} \in H^{1}(\Omega)$ be the solution of

$$
\Delta P U_{i}+\gamma(x) \cdot \nabla P U_{i}-P U_{i}+\varepsilon^{2} e^{U_{i}}=0 \quad \text { in } \Omega, \quad \frac{\partial P U_{i}}{\partial \nu}=0 \quad \text { on } \partial \Omega
$$

By standard regularity theory, $P U_{i} \in C^{\infty}(\bar{\Omega})$, so that $P U_{i}$ is indeed a classical solution of 3.9 .

Observe that each function $P U_{i}$ depends on $\varepsilon>0, d_{i}$ and $\zeta_{i}$, but for notational simplicity, we unify this dependence using the subindex $i$.

For every $i=1, \ldots, m$, consider the function $H_{i} \in C^{\infty}(\bar{\Omega})$ given by

$$
H_{i}(x):=P U_{i}(x)-U_{i}(x), \quad \text { for } \quad x \in \bar{\Omega} .
$$

From $3.9, H_{i}$ solves the equation

$$
\Delta H_{i}+\gamma(x) \cdot \nabla H_{i}-H_{i}=U_{i}-\gamma(x) \cdot \nabla U_{i} \quad \text { in } \Omega
$$

with the boundary condition

$$
\frac{\partial H_{i}}{\partial \nu}=-\frac{\partial U_{i}}{\partial \nu} \quad \text { on } \partial \Omega
$$

The following assumptions on the parameters $d_{i}$ and $\zeta_{i}$ will play a crucial role in what follows.

We assume that for every $i=1, \ldots, m$, the parameters $d_{i}>0$ and $\zeta_{i} \in \bar{\Omega}$ depend on $\varepsilon>0$. This dependence is expressed by the conditions:

$$
\lim _{\varepsilon \rightarrow 0^{+}} \varepsilon d_{i}^{\alpha}=0, \quad \forall \alpha>0
$$

and in the case that $\zeta_{i} \in \Omega$, for some $c_{0}>0$ and for some $\kappa \geq 1$

$$
\operatorname{dist}\left(\zeta_{i}, \partial \Omega\right) \geq c_{0}|\ln (\varepsilon)|^{-\kappa} .
$$


Next, lemma concerns the asymptotic behavior of the functions $H_{i}$ in terms of $d_{i}, \zeta_{i}$ and $\varepsilon>0$ small enough. For every fixed $i=1, \ldots, m$ and $\zeta_{i} \in \bar{\Omega}$, we will use the convention that

$$
c_{i}:=\left\{\begin{array}{l}
1, \quad \zeta_{i} \in \Omega \\
\frac{1}{2}, \zeta_{i} \in \partial \Omega .
\end{array}\right.
$$

Lemma 1 Assume conditions (3.12) and (3.13). Then, for every $i=1, \ldots, m$ and every $\varepsilon>0$ small enough, there exists a function $z_{i}$ such that

(i) for every $x \in \Omega$

$$
H_{i}(x)=-\ln \left(8 d_{i}^{2}\right)+c_{i} H\left(x, \zeta_{i}\right)+z_{i}(x),
$$

where $H=H_{a}$ is defined in (1.17) and

(ii) $\forall p \in(1,2), z_{i} \in W^{2, p}(\Omega) \cap C(\bar{\Omega})$ and

$$
\left\|z_{i}\right\|_{W^{2, p}(\Omega)}+\left\|z_{i}\right\|_{L^{\infty}(\Omega)} \leq C \varepsilon^{\frac{1}{p}} d_{i}^{\frac{1}{p}}
$$

where the constant $C>0$ depedens only on $p$.

Proof For notational simplicity, throughout this proof, we omit the subindex $i$. Let $\varepsilon>0$ be small, $d>0$ and $\zeta \in \bar{\Omega}$.

For $x \in \Omega$, we have that $H(x):=P U(x)-U(x)$. We set for $x \in \bar{\Omega}$

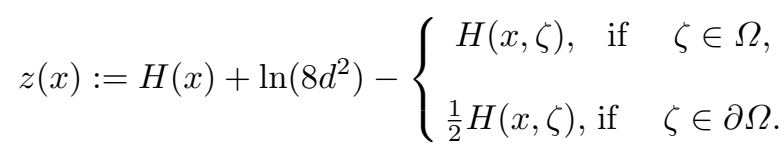

Recall from 1.15) that $\mathcal{L}=\Delta+\gamma(x) \cdot \nabla-1$. From 2.10) and 3.10), the equation for $z$ reads as

$$
\begin{gathered}
\mathcal{L} z(x)=2 \ln \left(\frac{|x-\zeta|^{2}}{\varepsilon^{2} d^{2}+|x-\zeta|^{2}}\right)+4 \gamma(x) \cdot \frac{(x-\zeta)}{|x-\zeta|^{2}} \cdot \frac{\varepsilon^{2} d^{2}}{\varepsilon^{2} d^{2}+|x-\zeta|^{2}} \quad \text { in } \Omega, \\
\frac{\partial z}{\partial \nu}=-4 \nu(x) \cdot \frac{(x-\zeta)}{|x-\zeta|^{2}} \cdot \frac{1}{\varepsilon^{2} d^{2}+|x-\zeta|^{2}} \quad \text { on } \partial \Omega .
\end{gathered}
$$

For any $p>1$, we estimate

$$
\begin{aligned}
\int_{\Omega}\left|\ln \left(\frac{|x-\zeta|^{2}}{\varepsilon^{2} d^{2}+|x-\zeta|^{2}}\right)\right|^{p} d x & \leq C \int_{0}^{2 \operatorname{diam}(\Omega)} r\left|\ln \left(\frac{r^{2}}{\varepsilon^{2} d^{2}+r^{2}}\right)\right|^{p} d r \\
& =C \varepsilon^{2} d^{2} \int_{0}^{\frac{2 \mathrm{diam}(\Omega)}{\varepsilon d}} r\left|\ln \left(1+\frac{1}{r^{2}}\right)\right|^{p} d r \\
& \leq C \varepsilon^{2} d^{2}\left(\int_{0}^{1} r\left|\ln \left(1+\frac{1}{r^{2}}\right)\right|^{p} d r+\int_{1}^{\infty} r^{1-2 p} d r\right) \\
& \leq C \varepsilon^{2} d^{2} .
\end{aligned}
$$


Hence, we obtain that

$$
\left\|\ln \left(\frac{|x-\zeta|^{2}}{\varepsilon^{2} d^{2}+|x-\zeta|^{2}}\right)\right\|_{L^{p}(\Omega)} \leq C \varepsilon^{\frac{2}{p}} d^{\frac{2}{p}}
$$

As for the second term, let us take $p \in(1,2)$, so that

$$
\begin{aligned}
\int_{\Omega}\left|\frac{\gamma(x) \cdot(x-\zeta)}{|x-\zeta|^{2}} \cdot \frac{\varepsilon^{2} d^{2}}{\varepsilon^{2} d^{2}+|x-\zeta|^{2}}\right|^{p} d x & \leq C \varepsilon^{2-p} d^{2-p} \int_{0}^{\frac{2 \operatorname{diam}(\Omega)}{\varepsilon d}} \frac{r^{1-p}}{\left(1+r^{2}\right)^{p}} d r \\
& \leq C \varepsilon^{2-p} d^{2-p}\left(\int_{0}^{1} \frac{r^{1-p}}{\left(1+r^{2}\right)^{p}} d r+\int_{1}^{\infty} r^{1-p} d r\right) \\
& \leq C \varepsilon^{2-p} d^{2-p}
\end{aligned}
$$

and therefore

$$
\left\|\frac{\gamma(x) \cdot(x-\zeta)}{|x-\zeta|^{2}} \cdot \frac{\varepsilon^{2} d^{2}}{\varepsilon^{2} d^{2}+|x-\zeta|^{2}}\right\|_{L^{p}(\Omega)} \leq C \varepsilon^{\frac{2}{p}-1} d^{\frac{2}{p}-1} .
$$

As for the boundary term, if $\zeta \in \Omega$ we use conditions 3.12 and $(3.13)$, to find that, provided $\varepsilon>0$ and $\varepsilon d$ are small enough

$$
\left|\frac{\nu(x) \cdot(x-\zeta)}{|x-\zeta|^{2}} \cdot \frac{\varepsilon^{2} d^{2}}{\varepsilon^{2} d^{2}+|x-\zeta|^{2}}\right| \leq \frac{C \varepsilon^{2} d^{2}}{|x-\zeta|^{3}} \leq C \varepsilon d, \quad \forall x \in \partial \Omega,
$$

On the other hand, if $\zeta \in \partial \Omega$, from Lemma (3), we estimate

$$
\left|\frac{\nu(x) \cdot(x-\zeta)}{|x-\zeta|^{2}} \cdot \frac{\varepsilon^{2} d^{2}}{\varepsilon^{2} d^{2}+|x-\zeta|^{2}}\right| \leq \frac{C \varepsilon^{2} d^{2}}{\varepsilon^{2} d^{2}+|x-\zeta|^{2}}, \quad \forall x \in \partial \Omega .
$$

Let us take $\delta>0$ small but fixed, so that

$$
\left|\frac{\nu(x) \cdot(x-\zeta)}{|x-\zeta|^{2}} \cdot \frac{\varepsilon^{2} d^{2}}{\varepsilon^{2} d^{2}+|x-\zeta|^{2}}\right| \leq \frac{C \varepsilon^{2} d^{2}}{\delta^{2}}, \quad \forall x \in \partial \Omega \cap B_{\delta}^{c}(\zeta)
$$

while for any $p>1$, we estimate

$$
\begin{aligned}
\int_{\partial \Omega \cap B_{\delta}(\zeta)}\left|\frac{\nu(x) \cdot(x-\zeta)}{|x-\zeta|^{2}} \cdot \frac{\varepsilon^{2} d^{2}}{\varepsilon^{2} d^{2}+|x-\zeta|^{2}}\right|^{p} d x & \leq C \varepsilon d \int_{0}^{\frac{\delta}{\varepsilon d}} \frac{1}{\left(1+s^{2}\right)^{p}}(\text { bs. } 17) \\
& \leq C \varepsilon d .
\end{aligned}
$$

We conclude that for any $\zeta \in \bar{\Omega}$

$$
\left\|\frac{\nu(x) \cdot(x-\zeta)}{|x-\zeta|^{2}} \cdot \frac{\varepsilon^{2} d^{2}}{\varepsilon^{2} d^{2}+|x-\zeta|^{2}}\right\|_{L^{p}(\partial \Omega)} \leq C \varepsilon^{\frac{1}{p}} d^{\frac{1}{p}} .
$$

Standard elliptic regularity implies that for any $p \in(1,2), z \in W^{2, p}(\Omega)$. The Sobolev embeddings in (2.1) together with estimates (3.15), (3.16) and 3.19, imply that

$$
\|z\|_{L^{\infty}(\Omega)}+\|z\|_{W^{2, p}(\Omega)} \leq C \varepsilon^{\frac{1}{p}} d^{\frac{1}{p}}
$$

and this concludes the proof of the lemma. 
Now we are in a position to set our approximation. Consider the parameters $d_{1}, \ldots, d_{m} \in \mathbb{R}_{+}$and $\zeta_{1}, \ldots, \zeta_{m} \in \bar{\Omega}$ satisfying 3.12 and 3.13 respectively. In addition, assume that

$$
\ln \left(8 d_{i}^{2}\right)=c_{i} H\left(\zeta_{i}, \zeta_{i}\right)+\sum_{j \neq i} c_{j} G\left(\zeta_{i}, \zeta_{j}\right), \quad \forall i=1, \ldots, m .
$$

We set as approximation the function

$$
u_{\varepsilon}(x):=\sum_{i=1}^{m} P U_{i}(x)=\sum_{i=1}^{m} U_{i}(x)+H_{i}(x), \quad \text { for } x \in \bar{\Omega} .
$$

Using the rescaling in $(3.2)$, we also set for every $y \in \Omega_{\varepsilon}$

$$
\begin{aligned}
v_{\varepsilon}(y) & :=4 \ln (\varepsilon)+u_{\varepsilon}(\varepsilon y) \\
& =4(1-m) \ln (\varepsilon)+\sum_{i=1}^{m} \ln \left(\frac{8 d_{i}^{2}}{\left(d_{i}^{2}+\left|y-\zeta_{i}^{\prime}\right|^{2}\right)^{2}}\right)+H_{i}(\varepsilon y),
\end{aligned}
$$

where we have denoted $\zeta_{i}^{\prime}:=\frac{\zeta_{i}}{\varepsilon}$.

For our subsequent developments, we introduce another condition on the numbers $d_{1}, \ldots, d_{m}$ and the points $\zeta_{1}, \ldots, \zeta_{m} \in \bar{\Omega}$. Consider the real number

$$
c_{\varepsilon}:=\min \left\{\left|\zeta_{i}-\zeta_{j}\right|: i, j=1, \ldots, m, \quad i \neq j\right\}
$$

which is well defined, positive and uniformly bounded above, since $\Omega$ is bounded. We assume in addition that

$$
\lim _{\varepsilon \rightarrow 0^{+}} \frac{c_{\varepsilon}}{\varepsilon d_{i}}=\infty, \quad \forall i=1, \ldots, m .
$$

Condition 3.24 means that as $\varepsilon \rightarrow 0^{+}$, the numbers $d_{i}$ might go to infinity, but at a rate that is controlled by the distance between the points $\zeta_{i}$.

Let us denote

$$
W:=e^{v_{\varepsilon}}, \quad S\left(v_{\varepsilon}\right):=\Delta v_{\varepsilon}+\varepsilon \gamma(\varepsilon y) \cdot \nabla v_{\varepsilon}-\varepsilon^{2}\left(v_{\varepsilon}-4 \ln (\varepsilon)\right)+e^{v_{\varepsilon}} .
$$

Next lemma provides the size of the error term $S\left(v_{\varepsilon}\right)$.

Lemma 2 Assume hypotheses in Lemma 1 and conditions 3.20 and (3.24). Then for any $\alpha, \beta \in(0,1)$, there exists $\varepsilon_{0}>0$ small such that for any $\varepsilon \in$ $\left(0, \varepsilon_{0}\right)$, there exists a function $\theta_{\varepsilon}(y)$ such that

$$
\left|\theta_{\varepsilon}(y)\right| \leq C \varepsilon^{3+\alpha}+C \varepsilon^{\beta} \sum_{i=1}^{m}\left|y-\zeta_{i}^{\prime}\right|^{\beta}, \quad \forall y \in \Omega_{\varepsilon}
$$

and

$$
W(y)=\sum_{i=1}^{m} \frac{8 d_{i}^{2}}{\left(\varepsilon^{2} d_{i}^{2}+\left|y-\zeta_{i}^{\prime}\right|\right)^{2}}\left(1+\theta_{\varepsilon}(y)\right), \quad \forall y \in \Omega_{\varepsilon} .
$$


Even more,

$$
\left|S\left(v_{\varepsilon}\right)(y)\right| \leq C \varepsilon^{\alpha} \sum_{i=1}^{m} \frac{1}{1+\left|y-\zeta_{i}^{\prime}\right|^{3}}, \quad \forall y \in \Omega .
$$

Proof We proceed as in the proof of expressions (20) and (21) in [11. For the sake of completeness we include the detailed computations.

We first prove (3.25). Let $\varepsilon>0$ be small. Observe that for any $i=1, \ldots, m$ and any $y \in \Omega_{\varepsilon}$

$$
\begin{aligned}
v_{\varepsilon}(y) & =4 \ln (\varepsilon)+U_{i}(\varepsilon y)+H_{i}(\varepsilon y)+\sum_{j \neq i}\left(U_{j}(\varepsilon y)+H_{j}(\varepsilon y)\right) \\
& =\ln \left(\frac{8 d_{i}^{2}}{\left(d_{i}^{2}+\left|y-\zeta_{i}^{\prime}\right|^{2}\right)^{2}}\right)+H_{i}(\varepsilon y)+\sum_{j \neq i}\left(U_{j}(\varepsilon y)+H_{j}(\varepsilon y)\right) .
\end{aligned}
$$

Using (3.14, we find that

$$
\begin{aligned}
v_{\varepsilon}(y)= & \ln \left(\frac{8 d_{i}^{2}}{\left(d_{i}^{2}+\left|y-\zeta_{i}^{\prime}\right|^{2}\right)^{2}}\right)-\ln \left(8 d_{i}^{2}\right)+c_{i} H\left(\varepsilon y, \zeta_{i}\right)+z_{i}(\varepsilon y)+ \\
& +\sum_{j \neq i}\left(\ln \left(\frac{8 d_{j}^{2}}{\left(\varepsilon^{2} d_{j}^{2}+\left|\varepsilon y-\zeta_{j}\right|^{2}\right)^{2}}\right)-\ln \left(8 d_{j}^{2}\right)+c_{j} H\left(\varepsilon y, \zeta_{j}\right)+z_{j}(\varepsilon y)\right) .
\end{aligned}
$$

Therefore, for any $\alpha \in(0,1)$,

$$
\begin{aligned}
& v_{\varepsilon}(y)=\ln \left(\frac{1}{\left(d_{i}^{2}+\left|y-\zeta_{i}^{\prime}\right|^{2}\right)^{2}}\right)+c_{i} H\left(\varepsilon y, \zeta_{i}\right) \\
& +\sum_{j \neq i}\left(\ln \left(\frac{1}{\left(\varepsilon^{2} d_{j}^{2}+\left|\varepsilon y-\zeta_{j}\right|^{2}\right)^{2}}\right)+c_{j} H\left(\varepsilon y, \zeta_{j}\right)\right)+\sum_{j=1}^{m} \mathcal{O}_{L^{\infty}\left(\Omega_{\varepsilon}\right)}\left(\varepsilon^{\alpha} d_{j}^{\alpha}\right) .
\end{aligned}
$$

To estimate more accurately (3.27), we first notice from Theorem 5 that for any fixed $j=1, \ldots, m$ and any $\beta \in(0,1)$,

$$
H\left(\varepsilon y, \zeta_{j}\right)=H\left(\zeta_{i}, \zeta_{j}\right)+\mathcal{O}_{L^{\infty}\left(\Omega_{\varepsilon}\right)}\left(\left|\varepsilon y-\zeta_{i}\right|^{\beta}\right), \quad \forall y \in \Omega_{\varepsilon} .
$$

Next, we consider two regimes: the first one close to the point $\zeta_{i}^{\prime}$ and the second one when we are far from $\zeta_{i}^{\prime}$. To be more precise, take any $\tilde{\alpha} \in(0,1)$ and $\delta_{\varepsilon} \in\left(0,(1-\tilde{\alpha}) c_{\varepsilon}\right]$, where $c_{\varepsilon}$ is defined in $[3.23)$. Notice that for any $j \neq i$ and any $y \in B_{\frac{\delta_{\varepsilon}}{\varepsilon}}\left(\zeta_{i}^{\prime}\right)$

$$
\left|y-\zeta_{j}^{\prime}\right| \geq \frac{\left|\zeta_{i}-\zeta_{j}\right|}{\varepsilon}-\left|y-\zeta_{i}^{\prime}\right| \geq \frac{\tilde{\alpha}\left|\zeta_{i}-\zeta_{j}\right|}{\varepsilon},
$$

so that $\left|\varepsilon y-\zeta_{j}\right| \geq \tilde{\alpha} c_{\varepsilon}$. 
Using conditions 3.12 and 3.24 , for any $y \in B_{\frac{\delta_{\varepsilon}}{\varepsilon}}\left(\zeta_{i}^{\prime}\right)$ and any $j \neq i$, we compute

$$
\begin{aligned}
\ln \left(\frac{1}{\left(\varepsilon^{2} d_{j}^{2}+\left|\varepsilon y-\zeta_{j}\right|^{2}\right)^{2}}\right) & =-4 \ln \left(\left|\varepsilon y-\zeta_{j}\right|\right)+\mathcal{O}_{L^{\infty}\left(B_{\frac{\delta_{\varepsilon}}{\varepsilon}}\left(\zeta_{i}^{\prime}\right)\right.}\left(\varepsilon^{2} d_{j}^{2}\left|y-\zeta_{j}^{\prime}\right|^{-2}\right) \\
& =-4 \ln \left(\left|\varepsilon y-\zeta_{j}\right|\right)+\mathcal{O}_{L^{\infty}\left(B_{\frac{\delta_{\varepsilon}}{\varepsilon}}\left(\zeta_{i}^{\prime}\right)\right.}\left(\varepsilon^{4} d_{j}^{4}\right) \\
& \left.=-4 \ln \left(\left|\zeta_{i}-\zeta_{j}\right|\right)+\mathcal{O}_{L^{\infty}\left(B_{\frac{\delta_{\varepsilon}}{\varepsilon}}\left(\zeta_{i}^{\prime}\right)\right.}\left(\varepsilon^{4-\tilde{\alpha}}+\varepsilon\left|y-\zeta_{i}^{\prime}\right|\right) \mid 3.29\right)
\end{aligned}
$$

Therefore, using expressions (3.28) and (3.29), we can choose $\tilde{\alpha}>1-\alpha$ such that for any $y \in B_{\frac{\delta_{\varepsilon}}{\varepsilon}}\left(\zeta_{i}^{\prime}\right)$ expression (3.27) reads as

$$
\begin{aligned}
v_{\varepsilon}(y)= & \ln \left(\frac{1}{\left(d_{i}^{2}+\left|y-\zeta_{i}^{\prime}\right|^{2}\right)^{2}}\right)+c_{i} H\left(\zeta_{i}, \zeta_{i}\right)+\sum_{j \neq i}\left(-4 \ln \left(\left|\zeta_{i}-\zeta_{j}\right|\right)+c_{j} H\left(\zeta_{i}, \zeta_{j}\right)\right) \\
& +\mathcal{O}_{L^{\infty}\left(B_{\frac{\delta_{\varepsilon}}{\varepsilon}\left(\zeta_{i}^{\prime}\right)}\right)}\left(\varepsilon^{4-\tilde{\alpha}}+\varepsilon d_{i}\left|y-\zeta_{i}^{\prime}\right|\right)+\mathcal{O}_{L^{\infty}\left(\Omega_{\varepsilon}\right)}\left(\left|\varepsilon y-\zeta_{i}\right|^{\beta}\right) \\
= & \ln \left(\frac{1}{\left(d_{i}^{2}+\left|y-\zeta_{i}^{\prime}\right|^{2}\right)^{2}}\right)+c_{i} H\left(\zeta_{i}, \zeta_{i}\right)+\sum_{j \neq i} c_{j} G\left(\zeta_{i}, \zeta_{j}\right) \\
& +\mathcal{O}_{L^{\infty}\left(B_{\frac{\delta_{\varepsilon}}{\varepsilon}}\left(\zeta_{i}^{\prime}\right)\right)}\left(\varepsilon^{3+\alpha}+\varepsilon^{\beta}\left|y-\zeta_{i}^{\prime}\right|^{\beta}\right) \\
= & \ln \left(\frac{8 d_{i}^{2}}{\left(d_{i}^{2}+\left|y-\zeta_{i}^{\prime}\right|^{2}\right)^{2}}\right)+\mathcal{O}_{L^{\infty}\left(B_{\frac{\delta_{\varepsilon}}{\varepsilon}}\left(\zeta_{i}^{\prime}\right)\right.}\left(\varepsilon^{3+\alpha}+\varepsilon^{\beta}\left|y-\zeta_{i}^{\prime}\right|^{\beta}\right) .
\end{aligned}
$$

Directly from the identities in 3.30 , we obtain that in $B_{\frac{\delta_{\varepsilon}}{\varepsilon}}\left(\zeta_{i}^{\prime}\right)$

$$
e^{v_{\epsilon}(y)}=\frac{8 d_{i}^{2}}{\left(d_{i}^{2}+\left|y-\zeta_{i}^{\prime}\right|^{2}\right)^{2}} e^{\mathcal{O}\left(\varepsilon^{3+\alpha}+\varepsilon^{\beta}\left|y-\zeta_{i}^{\prime}\right|^{\beta}\right)} .
$$

On the other hand, from (3.12) and (3.24), we can choose $\delta_{\varepsilon}$ such that for every $i=1, \ldots, m, \frac{\delta_{\varepsilon}}{\varepsilon d_{i}}$ is bounded below by $c \varepsilon^{1-\alpha} d_{i}^{1-\alpha}$. Hence, if $\left|y-\zeta_{i}^{\prime}\right| \geq \frac{\delta_{\varepsilon}}{\varepsilon}$, $\frac{\left|\varepsilon y-\zeta_{i}\right|}{\varepsilon d_{i}} \geq \frac{\delta_{\varepsilon}}{\varepsilon d_{i}}$ and from 3.22 we find that that

$$
\begin{aligned}
v_{\varepsilon}(y) & =4 \ln (\varepsilon)-\sum_{i=1}^{m}-4 \ln \left(\left|\varepsilon y-\zeta_{i}\right|\right)+\mathcal{O}_{L^{\infty}}\left(\varepsilon^{2} d_{i}^{2}\left|\varepsilon y-\zeta_{i}\right|^{-2}\right) \\
& =4 \ln (\varepsilon)-\sum_{i=1}^{m} 4 \ln \left(\left|\varepsilon y-\zeta_{i}\right|\right)+\mathcal{O}_{L^{\infty}(\Omega)}\left(\varepsilon^{\alpha} d_{i}^{\alpha}\right) \\
& =4 \ln (\varepsilon)+\mathcal{O}_{L^{\infty}(\Omega)}\left(\ln \left(\delta_{\varepsilon}\right)\right) .
\end{aligned}
$$

Hence, for $y \in \Omega_{\varepsilon}-\cup_{i=1}^{m} B_{\frac{\delta_{\varepsilon}}{\varepsilon}}\left(\zeta_{i}^{\prime}\right)$, we have that

$$
e^{v_{\varepsilon}(y)}=\mathcal{O}_{L^{\infty}\left(\Omega_{\varepsilon}-\cup_{i=1}^{m} B_{\frac{\varepsilon_{\varepsilon}}{\varepsilon}}\left(\zeta_{i}^{\prime}\right)\right)}\left(\varepsilon^{3+\alpha}\right) .
$$


Denoting by $\chi_{B}$ the characteristic function of a set $B \subset \mathbb{R}^{2}$, we write for $y \in \Omega_{\varepsilon}$

$$
\begin{aligned}
e^{v_{\varepsilon}(y)} & :=\sum_{i=1}^{m}\left(e^{v_{\varepsilon}(y)} \chi_{B_{\frac{\delta_{\varepsilon}}{\varepsilon}}\left(\zeta_{i}^{\prime}\right)}+e^{v_{\varepsilon}(y)} \chi_{\Omega_{\varepsilon}-\cup_{i=1}^{m} B \frac{\delta_{\varepsilon}}{\varepsilon}}\left(\zeta_{i}^{\prime}\right)\right. \\
& =\sum_{i=1}^{m} \frac{8 d_{i}^{2}}{\left(d_{i}^{2}+\left|y-\zeta_{i}^{\prime}\right|^{2}\right)^{2}} e^{\mathcal{O}\left(\varepsilon^{3+\alpha}+\left|\varepsilon y-\zeta_{i}\right|^{\beta}\right)}+\mathcal{O}\left(\varepsilon^{3+\alpha}\right)
\end{aligned}
$$

and asymptotics in 3.25 follow.

To find estimate (3.26), we use the fact that

$$
\begin{aligned}
S\left(v_{\varepsilon}(y)\right)= & e^{v_{\varepsilon}(y)}-\sum_{i=1}^{m} e^{4 \ln (\varepsilon)+U_{i}(\varepsilon y)} \\
= & \sum_{i=1}^{m}\left(e^{v_{\varepsilon}(y)}-e^{4 \ln (\varepsilon)+U_{i}(\varepsilon y)}\right) \chi_{B_{\frac{\delta_{\varepsilon}}{\varepsilon}}\left(\zeta_{i}^{\prime}\right)} \\
& +\left(e^{v_{\varepsilon}(y)}-\varepsilon^{4} \sum_{i=1}^{m} e^{U_{i}(\varepsilon y)}\right) \chi_{\Omega_{\varepsilon}-\cup_{i=1}^{m} B_{\frac{\delta_{\varepsilon}}{\varepsilon}}\left(\zeta_{i}^{\prime}\right)} \\
= & \sum_{i=1}^{m} \frac{8 d_{i}^{2}}{\left(d_{i}^{2}+\left|y-\zeta_{i}^{\prime}\right|^{2}\right)^{2}} \theta_{\varepsilon}(y)+\mathcal{O}\left(\varepsilon^{3+\alpha}\right)
\end{aligned}
$$

from where 3.26 follows.

\section{The reduction scheme}

In this part we use the approximation (3.21) described in section 3 to solve equation (1.11) using a finite dimensional reduction procedure.

We use the convention that $m=k+l$ for some $k, l \in\{0, \ldots, m\}$ and that

$$
\zeta_{1}, \ldots, \zeta_{k} \in \Omega \quad \text { and } \quad \zeta_{k+1}, \ldots, \zeta_{k+l} \in \partial \Omega
$$

It will be more convenient to work with the rescaling (3.2). Using the function described in 3.22 , we look for a solution $V_{\varepsilon}$ of equation (3.3) having the form

$$
V_{\varepsilon}(y):=v_{\varepsilon}(y)+\phi(y), \quad y \in \Omega_{\varepsilon}
$$

so that $\phi$ must solve the nonlinear boundary value problem

$$
\Delta \phi-\varepsilon^{2} \phi=-S\left(v_{\varepsilon}\right)-e^{v_{\varepsilon}} \phi-\varepsilon \gamma(\varepsilon y) \cdot \nabla \phi-N(\phi) \text { in } \Omega_{\varepsilon}
$$

with the boundary condition

$$
\frac{\partial \phi}{\partial \nu}=0 \quad \text { on } \quad \partial \Omega_{\varepsilon},
$$


where we have denoted

$$
N(\phi):=e^{v_{\varepsilon}}\left[e^{\phi}-1-\phi\right] .
$$

To solve 4.1)-4.2, we follow the developments from section 3 in [11.

For $i=1, \ldots, m$ fixed, we write

$$
J_{i}:=\left\{\begin{array}{l}
2, \quad \text { for } 1 \leq i \leq k \\
1, \text { for } k+1 \leq i \leq k+l .
\end{array}\right.
$$

Consider the following linear problem: given a function $h \in L^{\infty}\left(\Omega_{\varepsilon}\right)$, find $\phi \in C^{1}\left(\overline{\Omega_{\varepsilon}}\right)$ and constants $c_{i j}$ for $i=1, \ldots, m$ and for $j=1, J_{i}$ such that

$$
\Delta \phi-\varepsilon^{2} \phi=-e^{v_{\varepsilon}} \phi+h+\sum_{i=1}^{m} \sum_{j=1}^{J_{i}} c_{i j} \chi_{i j} Z_{i j} \quad \text { in } \quad \Omega_{\varepsilon}
$$

with the boundary and orthogonality conditions

$$
\frac{\partial \phi}{\partial \nu}=0 \quad \text { on } \quad \partial \Omega_{\varepsilon}, \quad \int_{\Omega_{\varepsilon}} \chi_{i j} Z_{i j} \phi=0, \quad \forall i=1, \ldots, m, \quad j=1, J_{i},
$$

where the functions $Z_{i j}, \chi_{i j}$ are next defined.

For $i=1, \ldots, m$, we set

$$
z_{i 0}(y):=\frac{1}{d_{i}}-\frac{2 d_{i}}{d_{i}^{2}+|y|^{2}}, \quad z_{i j}=\frac{y_{j}}{d_{i}^{2}+|y|^{2}}, \quad \forall j=1, J_{i}
$$

For any $i=1, \ldots, m$ fixed, Lemma 2.1 in 14 guarantees that the only solutions to

$$
\Delta \phi+\frac{8 d_{i}^{2}}{d_{i}^{2}+|y|^{2}} \phi=0 \quad \text { in } \mathbb{R}^{2}, \quad|\phi(y)| \leq C\left(1+|y|^{\sigma}\right), \quad \text { for some } \sigma>0
$$

are the linear combinations of $z_{i j}(y)$ for $j=0,1,2$. Observe that

$$
\frac{8 d_{i}^{2}}{d_{i}^{2}+|y|^{2}}=e^{V(y)}, \quad y \in \mathbb{R}^{2}
$$

where $V$ is the function in 3.5 with $d=d_{i}$ and $\zeta^{\prime}=0$.

Next, let $r_{0}>0$ be a large number and $\chi: \mathbb{R} \rightarrow[0,1]$ be a non-negative smooth cut-off function so that

$$
\chi(r)=\left\{\begin{array}{l}
1, \quad \text { if } \quad r \leq r_{0} \\
0, \text { if } \quad r \geq r_{0}+1 .
\end{array}\right.
$$

For $i=1, \ldots, k$, we have $\zeta_{i} \in \Omega$ and we define

$$
\chi_{i}(y):=\chi\left(\left|y-\zeta_{i}^{\prime}\right|\right), \quad Z_{i j}(y):=\chi_{i}(y) z_{i j}\left(y-\zeta_{i}^{\prime}\right), \quad j=1,2 .
$$


As for $i=k+1, \ldots, k+l$, we have that $\zeta_{i} \in \partial \Omega$. For notational simplicity, assume for the moment that $\zeta_{i}=0$ and that the inner unit normal vector to $\partial \Omega$ at $\zeta_{i}$ is the vector $\mathrm{e}_{2}=(0,1)$. Hence, there exists $r_{1}>0, \delta>0$ small and a function $\mathrm{p}:(-\delta, \delta) \rightarrow \mathbb{R}$ satisfying

$$
\mathrm{p} \in C^{\infty}(-\delta, \delta), \quad \mathrm{p}(0)=0, \quad \mathrm{p}^{\prime}(0)=0
$$

and such that

$$
\Omega \cap B_{r_{1}}(\zeta)=\left\{\left(x_{1}, x_{2}\right):-\delta<x_{1}<\delta, \quad \mathrm{p}\left(x_{1}\right)<x_{2}\right\} \cap B_{r_{1}}(0,0) .
$$

Consider the flattening change of variables $F_{i}: \Omega \cap B_{r_{1}}(\zeta) \rightarrow \mathbb{R}^{2}$ defined by

$F_{i}:=\left(F_{i 1}, F_{i 2}\right), \quad$ where $\quad F_{i 1}:=x_{1}+\frac{x_{2}-\mathrm{p}\left(\mathrm{x}_{1}\right)}{1+\left|\mathrm{p}^{\prime}\left(x_{1}\right)\right|^{2}} \mathrm{p}^{\prime}\left(x_{1}\right) \quad F_{i 2}:=x_{2}-\mathrm{p}\left(x_{1}\right)$.

At this point we remark that the radius $r_{1}$ must satisfy that $r_{1} \in\left(0, c_{\varepsilon}\right)$, where $c_{\varepsilon}$ is defined in 3.23 .

Throughout our discussions we will assume that $r_{1} \in\left(\frac{1}{2} c_{\varepsilon}, c_{\varepsilon}\right)$, so that $\frac{r_{1}}{\varepsilon} \rightarrow \infty$ as $\varepsilon \rightarrow 0$.

Recalling that $\zeta_{i}^{\prime}=\frac{\zeta_{i}}{\varepsilon}$, we set for $y \in \partial \Omega_{\varepsilon} \cap B \frac{r_{1}}{\varepsilon}\left(\zeta_{i}^{\prime}\right)$,

$$
F_{i}^{\varepsilon}(y):=\frac{1}{\varepsilon} F_{i}(\varepsilon y)
$$

and define

$$
\chi_{i}(y):=\chi\left(\left|F_{i}^{\varepsilon}(y)\right|\right), \quad Z_{i j}(y):=\chi_{i}(y) z_{i j}\left(F_{i}^{\varepsilon}(y)\right) .
$$

The following proposition accounts for the solvability of the linear problem (4.3)-(4.4). For this we define the following norm

$$
\|h\|_{*}:=\sup _{y \in \Omega_{\varepsilon}} \frac{|h(y)|}{\varepsilon^{2}+\sum_{i=1}^{m}\left(1+\left|y-\zeta_{i}^{\prime}\right|\right)^{-2-\sigma}} .
$$

Proposition 2 Assume the conditions of Lemma 2 on the parameters $d_{i}$ and $\zeta_{i}$. For any $\varepsilon>0$ small enough and any given $h \in L^{\infty}\left(\Omega_{\varepsilon}\right)$ there exists $\phi \in$ $C^{1}\left(\overline{\Omega_{\varepsilon}}\right)$ and constants $c_{i j} \in \mathbb{R}$ where $\phi$ is the unique solution of $(4.3)-(4.4)$. Moreover, there exists a constant $C>0$ independent of $\varepsilon>0$ such that

$$
\|\nabla \phi\|_{L^{\infty}\left(\Omega_{\varepsilon}\right)}+\|\phi\|_{L^{\infty}\left(\Omega_{\varepsilon}\right)} \leq C \ln \left(\frac{1}{\varepsilon}\right)\|h\|_{*} .
$$


With our choice of the radius $r_{1}$, the proof of Proposition 2 follows the same lines of the proof of Proposition 3.1 in [11] with only slight changes.

We do comment on the presence of the $|\ln (\varepsilon)|$ term in the statement of Proposition 2 For any $i=1, \ldots, m, Z_{i 0}$ is an approximate element of the kernel of 4.3- 4.4 . Hence any solution $\phi$ of 4.3 -4.4 can be written at main order as $\tilde{\phi}+\sum_{i=1}^{m} c_{i 0} \chi_{i} Z_{i 0}$, with $c_{i 0}$ chosen so that $\tilde{\phi}$ satisfies (4.4) for $i=0, \ldots, m$. From the proof of Proposition 3.1 (see Lemma 3.4) in [11], we have that for any $i=1, \ldots, m$,

$$
c_{i 0}=\mathcal{O}\left(\ln \left(\frac{1}{\varepsilon}\right)\|h\|_{*}\right),
$$

while elliptic estimates (see Lemma 3.2 in [1]) allow us to control $\|\phi\|_{L^{\infty}\left(\Omega_{\varepsilon}\right)}$ uniformly in $\varepsilon>0$. We leave details to the reader.

Denote by $\zeta:=\left(\zeta_{1}, \ldots, \zeta_{m}\right)$ and $T(h)=\phi$ the linear operator given by Proposition 2. Clearly $T(h)$ depends on $\boldsymbol{\zeta}$, so we write

$$
T(h)=T(h)(\boldsymbol{\zeta}) .
$$

Proceeding exactly as in section 3 in $[9]$, we obtain that the mapping $\boldsymbol{\zeta} \rightarrow$ $T(h)(\boldsymbol{\zeta})$ is differentiable in $\boldsymbol{\zeta}$ and

$$
\left\|D_{\zeta} T(h)\right\| \leq C \ln \left(\frac{1}{\varepsilon}\right)^{2}\|h\|_{*} .
$$

As a direct application of Proposition 2 and a fixed point argument, we solve the nonlinear problem

$\Delta \phi-\varepsilon^{2} \phi=-S\left(v_{\varepsilon}\right)-e^{v_{\varepsilon}} \phi-\varepsilon \gamma(\varepsilon y) \cdot \nabla \phi-N(\phi)+\sum_{i=1}^{m} \sum_{j=1, J_{i}} c_{i j} \chi_{i j} Z_{i j} \quad$ in $\quad \Omega_{\varepsilon}$

with the boundary and orthogonality conditions in 4.4.

Proposition 3 Under assumptions in Propososition 2, given any $\alpha>0$ for every $\varepsilon>0$ small there exists a solution $\phi$ and constants $c_{i j} \in \mathbb{R}^{N}$ satisfying (4.6) - (4.4) and such that

$$
\|\nabla \phi\|_{L^{\infty}\left(\Omega_{\varepsilon}\right)}+\|\phi\|_{L^{\infty}\left(\Omega_{\varepsilon}\right)} \leq C \varepsilon^{\alpha} \ln \left(\frac{1}{\varepsilon}\right) .
$$

Even more, $\phi=\Phi(\boldsymbol{\zeta})$ is differentiable respect to $\boldsymbol{\zeta}$ and

$$
\left\|D_{\zeta} \Phi\right\| \leq C \varepsilon^{\alpha} \ln \left(\frac{1}{\varepsilon}\right)^{2}
$$

Proof We proceed using a fixed point argument, as in the proof of Lemma 4.1 in 11$]$ using the fact that

$$
\|\nabla \phi\|_{*} \leq C\|\nabla \phi\|_{L^{\infty}\left(\Omega_{\varepsilon}\right)}
$$




\section{The energy estimate}

Our next step, consists in choosing the points $\zeta_{i} \in \bar{\Omega}$ so that the real numbers, (actually functions of $\left.\zeta:=\left(\zeta_{1}, \ldots, \zeta_{m}\right)\right) c_{i j}$, in equation 4.6), are identically zero. Thus leading to the solution of 4.1)- 4.2 .

Notice that the dimension of the approximate kernel of the linear problem (4.3)-(4.4) is $3 k+2 l$. We get rid of $m=k+l$ elements of this approximate kernel by choosing parameters $d_{1}, \ldots, d_{m}$ satisfying $(3.20)$. On the other hand, associated to the points $\zeta_{i} \in \Omega$, we must get rid of the constants $c_{i 1}$ and $c_{i 2}$, while for points $\zeta_{i} \in \partial \Omega$, we must get rid of only the constants $c_{i 1}$.

Throughout this part, we take $\zeta_{1}, \ldots, \zeta_{m} \in \bar{\Omega}$ satisfying conditions 3.13 and (3.24). In addition, we assume that for some $\kappa \geq 1$

$$
c|\ln (\varepsilon)|^{-\kappa} \leq\left|\zeta_{i}-\zeta_{j}\right| \leq C, \quad \forall i, j=1, \ldots, m, \quad i \neq j .
$$

Since

$$
c_{\varepsilon}:=\min \left\{\left|\zeta_{i}-\zeta_{j}\right|: i, j=1, \ldots, m, \quad i \neq j\right\}
$$

we notice that $c_{\varepsilon} \geq c|\ln (\varepsilon)|^{-\kappa}$.

Since the approximation in 3.21 depends on the points $\zeta_{i}$, we write

$$
u_{\varepsilon}(\boldsymbol{\zeta}):=u_{\varepsilon}=\sum_{i=1}^{m} U_{i}+H_{i}, \quad \text { in } \quad \bar{\Omega},
$$

where

$$
U_{i}(x):=\ln \left(\frac{8 d_{i}^{2}}{\left(\varepsilon^{2} d_{i}^{2}+\left|x-\zeta_{i}\right|^{2}\right)^{2}}\right), \quad \text { for } x \in \mathbb{R}^{2}
$$

and the $d_{i}$ 's are real numbers satisfying

$$
\ln \left(8 d_{i}^{2}\right)=c_{i} H\left(\zeta_{i}, \zeta_{i}\right)+\sum_{j \neq i} c_{j} G\left(\zeta_{i}, \zeta_{j}\right), \quad \forall i=1, \ldots, m .
$$

Denote also $\phi=\phi(\boldsymbol{\zeta})$ and $c_{i j}(\boldsymbol{\zeta})$ the unique solution of 4.2 - 4.6 and finally set $\tilde{\phi}(\boldsymbol{\zeta}):=\phi(\boldsymbol{\zeta})\left(\frac{x}{\varepsilon}\right)$.

For any $u \in H^{1}(\Omega)$, we consider the energy

$$
J(u):=\frac{1}{2} \int_{\Omega} a(x)\left(|\nabla u|^{2}+u^{2}\right) d x-\varepsilon^{2} \int_{\Omega} a(x) e^{u}
$$

which is well defined due to the Moser-Trudinger inequality. It is well known that critical points of $J$ are nothing but solutions of equation (1.11). Let us introduce the so-called reduced energy

$$
\mathcal{F}_{\varepsilon}(\boldsymbol{\zeta}):=J\left(u_{\varepsilon}(\boldsymbol{\zeta})+\tilde{\phi}(\boldsymbol{\zeta})\right)
$$

The aim of the next result is to understand the role played by $\mathcal{F}_{\varepsilon}$ and its asymptotic behavior as $\varepsilon \rightarrow 0$. 
Proposition 4 (i) If $\boldsymbol{\zeta}=\left(\zeta_{1}, \ldots, \zeta_{m}\right)$, with the $\zeta_{i}$ 's satisfying conditions (3.13), 3.24 and (5.1), is a critical point of the functional $\mathcal{F}_{\varepsilon}$ then $u=$ $u_{\varepsilon}(\boldsymbol{\zeta})+\phi(\boldsymbol{\zeta})$ is a critical point of $J$, i.e. a solution of equation (1.11).

(ii) There holds true that

$$
\mathcal{F}_{\varepsilon}(\boldsymbol{\zeta})=\mathcal{F}_{0}(\boldsymbol{\zeta})+\tilde{\theta}_{\varepsilon}(\boldsymbol{\zeta})
$$

$C^{1}$-uniformly in $\boldsymbol{\zeta}=\left(\zeta_{1}, \ldots, \zeta_{m}\right)$. Here (given $c_{0}:=-4 \pi(2-\ln 8)$ )

$$
\begin{aligned}
\mathcal{F}_{0}(\boldsymbol{\zeta}):= & \sum_{i=1}^{m} a\left(\zeta_{i}\right)\left(c_{0}+8 \pi|\ln \varepsilon|\right)-4 \pi \sum_{i=1}^{m} a\left(\zeta_{i}\right)\left[H\left(\zeta_{i}, \zeta_{i}\right)+\sum_{j \neq i} G\left(\zeta_{i}, \zeta_{j}\right)\right] \\
& \text { if } \zeta_{1}, \ldots, \zeta_{m} \in \Omega
\end{aligned}
$$

and

$$
\begin{aligned}
\mathcal{F}_{0}(\boldsymbol{\zeta}):= & \sum_{i=1}^{m} a\left(\zeta_{i}\right)\left(c_{0}+8 \pi|\ln \varepsilon|\right)-2 \pi \sum_{i=1}^{m} a\left(\zeta_{i}\right)\left[H\left(\zeta_{i}, \zeta_{i}\right)+\sum_{j \neq i} G\left(\zeta_{i}, \zeta_{j}\right)\right] \\
& \text { if } \zeta_{1}, \ldots, \zeta_{m} \in \partial \Omega
\end{aligned}
$$

Moreover $\tilde{\theta}_{\varepsilon}$ is a $C^{1}$ - function such that $\left|\tilde{\theta}_{\varepsilon}\right|+\left|\nabla_{\zeta} \tilde{\theta}_{\varepsilon}\right| \rightarrow 0$ as $\varepsilon \rightarrow 0$,

Proof First of all, we prove $(i)$ and the fact that there exists a $C^{1}$ differentiable function $\tilde{\theta}_{\varepsilon}$ such that

$$
\mathcal{F}_{\varepsilon}(\boldsymbol{\zeta})=J\left(u_{\varepsilon}(\boldsymbol{\zeta})\right)+\tilde{\theta}_{\varepsilon}(\boldsymbol{\zeta})
$$

and as $\varepsilon \rightarrow 0$,

$$
\left|\tilde{\theta}_{\varepsilon}\right|+\left|\nabla_{\zeta} \tilde{\theta}_{\varepsilon}\right| \rightarrow 0
$$

uniformly in $\zeta=\left(\zeta_{1}, \ldots, \zeta_{m}\right)$. The proof follows the same lines of Lemma 5.1 and Lemma 5.2 in $[1]$, with no changes. We leave details to the reader. We also refer the reader to 32,33 for similar computations.

Then we analyze the asymptotic behavior of the energy $J\left(u_{\varepsilon}(\boldsymbol{\zeta})\right)$. We only consider the case when the points belong to $\Omega$, because the proof when the points lie on the boundary $\partial \Omega$ relies on similar arguments. Moreover, we only compute the $C^{0}$-expansion because the $C^{1}$-estimate can be obtained in a similar way.

First of all, since for every $i=1, \ldots, m$

$$
\begin{gathered}
\operatorname{div}\left(a(x) \nabla P U_{i}\right)-a(x) P U_{i}+\varepsilon^{2} a(x) e^{U_{i}}=0 \quad \text { in } \Omega \\
\frac{\partial P U_{i}}{\partial \nu}=0 \quad \text { on } \quad \partial \Omega
\end{gathered}
$$

we know that for any $j, i=1, \ldots, m$,

$$
\int_{\Omega} a(x)\left(\nabla P U_{i} \cdot \nabla P U_{j}+P U_{i} \cdot P U_{j}\right) d x=\varepsilon^{2} \int_{\Omega} a(x) e^{U_{i}} P U_{j} d x
$$


Hence, we compute

$$
\begin{aligned}
J\left(u_{\varepsilon}(\boldsymbol{\zeta})\right)= & \frac{1}{2} \int_{\Omega} a(x)\left(\left|\sum_{i=1}^{m} \nabla P U_{i}\right|^{2}+\left|\sum_{i=1}^{m} P U_{i}\right|^{2}\right)-\varepsilon^{2} \int_{\Omega} a(x) e^{\sum_{i=1}^{m} P U_{i}} \\
= & \frac{1}{2} \sum_{i=1}^{m} \int_{\Omega} a(x)\left(\left|\nabla P U_{i}\right|^{2}+\left|P U_{i}\right|^{2}\right) \\
& +\frac{1}{2} \sum_{i, j=1 i \neq j}^{m} \int_{\Omega} a(x)\left(\nabla P U_{i} \cdot \nabla P U_{j}+P U_{i} \cdot P U_{j}\right)-\varepsilon^{2} \int_{\Omega} a(x) e^{\sum_{i=1}^{m} P U_{i}} \\
= & \underbrace{\sum_{i=1}^{m} \frac{\varepsilon^{2}}{2} \int_{\Omega} a(x) e^{U_{i}} P U_{i} d x}_{I}+\underbrace{\sum_{i, j i \neq j} \frac{\varepsilon^{2}}{2} \int_{\Omega} a(x) e^{U_{i}} P U_{j} d x}_{I I}-\underbrace{\varepsilon^{2} \int_{\Omega} a(x) e^{\sum_{i=1}^{m} P U_{i}} d x}_{I I I} .
\end{aligned}
$$

We first compute $I$. Fix $i=1, \ldots, m$ so that

$$
\varepsilon^{2} \int_{\Omega} a(x) e^{U_{i}} P U_{i} d x=\int_{\Omega} a(x) \frac{8 \varepsilon^{2} d_{i}^{2}}{\left(\varepsilon^{2} d_{i}^{2}+\left|x-\zeta_{i}\right|^{2}\right)^{2}}\left(U_{i}(x)+H_{i}(x)\right) d x .
$$

From Lemma 1, for any $\alpha \in(0,1)$

$$
U_{i}(x)+H_{i}(x)=\ln \left(\frac{1}{\left(\varepsilon^{2} d_{i}^{2}+\left|x-\zeta_{i}\right|^{2}\right)^{2}}\right)+H\left(x, \zeta_{i}\right)+\mathcal{O}\left(\varepsilon^{\alpha}\right), \quad x \in \Omega .
$$

Using the change of variables $x=\zeta_{i}+\varepsilon d_{i} y$, we obtain

$$
\begin{aligned}
& \varepsilon^{2} \int_{\Omega} a(x) e^{U_{i}} P U_{i} d x \\
& =\int_{\Omega_{\varepsilon d_{i}-\frac{\zeta_{i}}{\varepsilon d_{i}}}} a\left(\zeta_{i}+\varepsilon d_{i} y\right) \frac{8}{\left(1+|y|^{2}\right)^{2}}\left(-\ln \left(\varepsilon^{4} d_{i}^{4}\right)+\ln \left(\frac{1}{\left(1+|y|^{2}\right)^{2}}\right)+H\left(\zeta_{i}+\varepsilon d_{i} y, \zeta_{i}\right)+\mathcal{O}\left(\varepsilon^{\alpha}\right)\right) d y \\
& =\int_{\Omega_{\varepsilon d_{i}-\frac{\zeta_{i}}{\varepsilon d_{i}}}} a\left(\zeta_{i}\right) \frac{8}{\left(1+|y|^{2}\right)^{2}}\left(-\ln \left(\varepsilon^{4} d_{i}^{4}\right)+\ln \left(\frac{1}{\left(1+|y|^{2}\right)^{2}}\right)+H\left(\zeta_{i}, \zeta_{i}\right)+\mathcal{O}\left(\varepsilon^{\alpha} d_{i}^{\alpha}|y|^{\alpha}\right)\right) d y \\
& =\int_{\mathbb{R}^{2}} a\left(\zeta_{i}\right) \frac{8}{\left(1+|y|^{2}\right)^{2}}\left(-\ln \left(\varepsilon^{4} d_{i}^{4}\right)+\ln \left(\frac{1}{\left(1+|y|^{2}\right)^{2}}\right)+H\left(\zeta_{i}, \zeta_{i}\right)\right) d y+\mathcal{O}\left(\varepsilon^{\alpha} d_{i}^{\alpha}\right) .
\end{aligned}
$$

Since

$\int_{\mathbb{R}^{2}} \frac{8}{\left(1+|y|^{2}\right)^{2}} d y=8 \pi \quad$ and $\quad \int_{\mathbb{R}^{2}} \frac{8}{\left(1+|y|^{2}\right)^{2}} \ln \left(\frac{1}{\left(1+|y|^{2}\right)^{2}}\right) d y=-16 \pi$,

we find that

$$
I=-8 \pi \sum_{i=1}^{m} a\left(\zeta_{i}\right)\left(\ln \left(\varepsilon^{2} d_{i}^{2}\right)+1\right)+4 \pi \sum_{i=1}^{m} a\left(\zeta_{i}\right) H\left(\zeta_{i}, \zeta_{i}\right)+\mathcal{O}\left(\varepsilon^{\alpha}\right) .
$$


Here we also used the fact that the parameters $d_{i}$ 's satisfy $\frac{1}{C} \leq d_{i} \leq|\ln (\varepsilon)|^{C}$ and $\lim _{\varepsilon \rightarrow 0^{+}} \varepsilon d_{i}=0$ (as it follows directly from [2.5) and Proposition 1 and expression and (3.20) $)$.

Next, we compute $I I$. First we notice for $i \neq j$ that

$$
\begin{gathered}
\varepsilon^{2} \int_{\Omega} a(x) e^{U_{i}} P U_{j} d x \\
=\int_{\Omega} a(x) \frac{8 \varepsilon^{2} d_{i}^{2}}{\left(\varepsilon^{2} d_{i}^{2}+\left|x-\zeta_{i}\right|^{2}\right)^{2}}\left(\ln \left(\frac{1}{\left(\varepsilon^{2} d_{j}^{2}+\left|x-\zeta_{j}\right|^{2}\right)^{2}}\right)+H\left(x, \zeta_{j}\right)+\mathcal{O}\left(\varepsilon^{\alpha} d_{j}^{\alpha}\right)\right) d y .
\end{gathered}
$$

Recall that

$$
c_{\varepsilon}:=\min \left\{\left|\zeta_{i}-\zeta_{j}\right|: i, j=1, \ldots, m, \quad i \neq j\right\} .
$$

On the other hand, for $\left|x-\zeta_{j}\right| \geq c_{\varepsilon}$, we have that

$$
\begin{aligned}
\ln \left(\frac{1}{\left(\varepsilon^{2} d_{j}^{2}+\left|x-\zeta_{j}\right|^{2}\right)^{2}}\right) & =-4 \ln \left(\left|x-\zeta_{j}\right|\right)+\mathcal{O}_{L^{\infty}\left(B_{c_{\varepsilon}}\left(\zeta_{i}\right)\right)}\left(\varepsilon^{2} d_{j}^{2}\left|y-\zeta_{j}^{\prime}\right|^{-2}\right) \\
& =-4 \ln \left(\left|\varepsilon y-\zeta_{j}\right|\right)+\mathcal{O}_{L^{\infty}\left(B \frac{c_{\varepsilon}}{\varepsilon}\left(\zeta_{i}^{\prime}\right)\right)}\left(\varepsilon^{2} d_{j}^{2}\left|y-\zeta_{j}^{\prime}\right|^{-2}\right) \\
& =-4 \ln \left(\left|\zeta_{i}-\zeta_{j}\right|\right)+\mathcal{O}_{\left.L_{\left(B \frac{c_{\varepsilon}}{\varepsilon}\right.}\left(\zeta_{i}^{\prime}\right)\right)}\left(\varepsilon^{\alpha}\right)
\end{aligned}
$$

Also

$$
H\left(x, \zeta_{j}\right)=H\left(\zeta_{i}, \zeta_{j}\right)+\mathcal{O}_{L^{\infty}(\Omega)}\left(\left|x-\zeta_{j}\right|^{\alpha}\right), \quad \forall x \in \Omega
$$

so that

$$
\begin{aligned}
\varepsilon^{2} \int_{\Omega} a(x) e^{U_{i}} P U_{j} d x & =\int_{\Omega} a\left(\zeta_{i}\right) \frac{8}{\left(1+|y|^{2}\right)^{2}}\left(-4 \ln \left(\left|\zeta_{i}-\zeta_{j}\right|\right)+H\left(\zeta_{i}, \zeta_{j}\right)+\mathcal{O}\left(\varepsilon^{\alpha}\right)\right) d y \\
& =8 \pi a\left(\zeta_{i}\right) G\left(\zeta_{i}, \zeta_{j}\right)+\mathcal{O}\left(\varepsilon^{\alpha}\right)
\end{aligned}
$$

Therefore,

$$
I I=\sum_{i, j, i \neq j}^{m} 4 \pi a\left(\zeta_{i}\right) G\left(\zeta_{i}, \zeta_{j}\right)+\mathcal{O}\left(\varepsilon^{\alpha}\right) .
$$

Finally, we compute $I I I$. To do this we appeal to Lemma 2 to obtain that for fix $i=1, \ldots, m$ and for any $x \in B_{c_{\varepsilon}}\left(\zeta_{i}\right)$

$$
\begin{aligned}
I I I & =\varepsilon^{2} \int_{\Omega} a(x) e^{\sum_{j=1}^{m} U_{j}(x)+H_{j}(x)} d x \\
& =\varepsilon^{2} \sum_{i=1}^{m} \int_{\Omega} a(x) e^{\sum_{j=1}^{m} U_{j}(x)+H_{j}(x)} \chi_{B_{c_{\varepsilon}}\left(\zeta_{i}\right)} d x+\varepsilon^{2} \int_{\Omega} a(x) e^{\sum_{j=1}^{m} U_{j}(x)+H_{j}(x)} \chi_{\Omega-\cup_{i=1}^{m} B_{c_{\varepsilon}}\left(\zeta_{i}\right)} d x \\
& =8 \pi \sum_{i=1}^{m} a\left(\zeta_{i}\right)+\mathcal{O}\left(\varepsilon^{\alpha}\right)
\end{aligned}
$$


Finally putting together estimates (5.8), 5.9 and (5.10) we obtain that

$J\left(u_{\varepsilon}(\boldsymbol{\zeta})\right)=-8 \pi \sum_{i=1}^{m} a\left(\zeta_{i}\right)\left(2+\ln \left(\varepsilon^{2} d_{i}^{2}\right)\right)+4 \pi \sum_{i=1}^{m} a\left(\zeta_{i}\right)\left[H\left(\zeta_{i}, \zeta_{i}\right)+\sum_{j \neq i} G\left(\zeta_{i}, \zeta_{j}\right)\right]+\mathcal{O}\left(\varepsilon^{\alpha}\right)$

and using condition 5.2 the desired estimate follows.

\section{Proofs of Theorems}

Proof (Proof of Theorem 1.) Let $\mathbf{s}:=\left(s_{1}, \ldots, s_{m}\right) \in(\partial \Omega)^{m}$ and $\mathbf{t}:=\left(t_{1}, \ldots, t_{m}\right) \in$ $\left(\mathbb{R}_{+}\right)^{m}$. Let us consider the configuration space

$$
\Lambda_{\delta}:=\left\{(\mathbf{s}, \mathbf{t}) \in(\partial \Omega)^{m} \times\left(\mathbb{R}_{+}\right)^{m}:\left|s_{i}-s_{j}\right| \geq \delta, \delta<t_{i}<1 / \delta\right\},
$$

for some $\delta>0$ small, independent of $\varepsilon>0$.

For any point in $(\mathbf{s}, \mathbf{t}) \in \Lambda_{\delta}$, we set

$$
\zeta_{i}:=s_{i}+|\ln (\varepsilon)|^{-1} t_{i} \nu\left(s_{i}\right), \quad \forall i=1, \ldots, m .
$$

Observe that 3.12, 3.13, 3.20 and 3.24 hold true. By Proposition 4 we have to find a critical point $\zeta^{\varepsilon}:=\left(\zeta_{1}^{\varepsilon}, \ldots, \zeta_{m}^{\varepsilon}\right) \in \Omega \times \cdots \times \Omega$ of the reduced energy $\mathcal{F}_{\varepsilon}$ where each $\zeta_{i}^{\varepsilon}$ is as in 6.1 . If we use the parametrization of the points $\zeta_{i}$ given in (6.1), we are lead to find a critical point $\left(\mathbf{s}^{\varepsilon}, \mathbf{t}^{\varepsilon}\right)$ of the reduced energy $\mathcal{F}_{\varepsilon}(\mathbf{s}, \mathbf{t})$.

By (5.5) and the property of Robin's function we deduce that $\mathcal{F}_{\varepsilon}$ reduces to

$\mathcal{F}_{\varepsilon}(\mathbf{s}, \mathbf{t})=8 \pi|\ln \varepsilon|\left[\sum_{i=1}^{m} a\left(s_{i}\right)+\Upsilon_{\varepsilon}(\mathbf{s})\right]+16 \pi\left[\sum_{i=1}^{m} a\left(s_{i}\right) \ln t_{i}+t \partial_{\nu} a\left(s_{i}\right)\right]+\Theta_{\varepsilon}(\mathbf{s}, \mathbf{t})$,

where the smooth functions $\Upsilon_{\varepsilon}(\mathbf{s})$ only depends on $\mathbf{s}$ while $\Theta_{\varepsilon}(\mathbf{s}, \mathbf{t})$ depends on both $\mathbf{s}$ and $\mathbf{t}$ and they are higher order terms, namely $\left|\Upsilon_{\varepsilon}\right|,\left|\nabla \Upsilon_{\varepsilon}\right|,\left|\Theta_{\varepsilon}\right|,\left|\nabla \Theta_{\varepsilon}\right| \rightarrow$ 0 as $\varepsilon \rightarrow 0$. The proof of this claim is postponed to the end.

Once the estimate 6.2 is proved, the claim easily follows by degree theory. Indeed, using 6.2, let us introduce the continuous functions

$$
\begin{aligned}
\frac{\nabla_{s_{i}} \mathcal{F}_{\varepsilon}(\mathbf{s}, \mathbf{t})}{|\ln \varepsilon|} & =\underbrace{8 \pi \nabla a\left(s_{i}\right)}_{:=\mathcal{S}_{i}\left(s_{i}\right)}+o(1), i=1, \ldots, m \\
\nabla_{t_{i}} \mathcal{F}_{\varepsilon}(\mathbf{s}, \mathbf{t}) & =\underbrace{16 \pi\left[\frac{a\left(s_{i}\right)}{t_{i}}+\partial_{\nu} a\left(s_{i}\right)\right]}_{:=\mathcal{T}_{i}\left(s_{i}, t_{i}\right)}+o(1), i=1, \ldots, m .
\end{aligned}
$$

Under assumption (A1), we know that $a$ has $m$ different strict local minima or local maxima points $\zeta_{1}^{*}, \ldots, \zeta_{m}^{*} \in \partial \Omega$. Set $s_{i}^{*}:=\zeta_{i}^{*}$. Therefore, for some $\rho>0$ 
small enough, the Brouwer degree $\operatorname{deg}\left(\nabla a, B\left(s_{i}^{*}, \rho\right), 0\right)$ is well defined and (see, for example, Corollary 2 in [1])

$$
\operatorname{deg}\left(\mathcal{S}_{i}, B\left(s_{i}^{*}, \rho\right), 0\right)= \pm 1 \neq 0 \text { for any } i=1, \ldots, m .
$$

On the other hand, since $\partial_{\nu} a\left(\zeta_{i}^{*}\right)<0$, we can choose $\rho$ small enough so that for any $s_{i} \in B\left(s_{i}^{*}, \rho\right)$ there exists a unique $t_{i}=t_{i}\left(s_{i}\right)=-\frac{a\left(s_{i}\right)}{\partial_{\nu} a\left(s_{i}\right)}$ such that $\mathcal{T}_{i}\left(s_{i}, t_{i}\right)=0$.

Set $t_{i}^{*}:=t_{i}\left(s_{i}^{*}\right)$ and let $\rho$ smaller if necessary, so that the Brouwer degree

$$
\operatorname{deg}\left(\left(\mathcal{S}_{i}, \mathcal{T}_{i}\right), B\left(s_{i}^{*}, \rho\right) \times B\left(t_{i}^{*}, \rho\right), 0\right)
$$

is well defined. Since $\partial_{t_{i}} \mathcal{T}_{i}\left(s_{i}, t_{i}\left(s_{i}\right)\right)<0$, by (6.5) (see, for example, Lemma 5.7 in [24]) we immediately get that

$$
\operatorname{deg}\left(\left(\mathcal{S}_{i}, \mathcal{T}_{i}\right), B\left(s_{i}^{*}, \rho\right) \times B\left(t_{i}^{*}, \rho\right), 0\right) \neq 0 \text { for any } i=1, \ldots, m .
$$

Finally, by 6.6 using the properties of Brouwer degree, we get

$$
\begin{aligned}
& \operatorname{deg}\left(\left(\mathcal{S}_{1}, \mathcal{T}_{1}\right), \ldots,\left(\mathcal{S}_{m}, \mathcal{T}_{m}\right),\left(B\left(s_{1}^{*}, \rho\right) \times B\left(t_{1}^{*}, \rho\right)\right) \times \cdots \times\left(B\left(s_{m}^{*}, \rho\right) \times B\left(t_{m}^{*}, \rho\right)\right), 0\right) \\
& =\operatorname{deg}\left(\left(\mathcal{S}_{1}, \mathcal{T}_{1}\right), B\left(s_{1}^{*}, \rho\right) \times B\left(t_{1}^{*}, \rho\right), 0\right) \times \cdots \times \operatorname{deg}\left(\left(\mathcal{S}_{m}, \mathcal{T}_{m}\right), B\left(s_{m}^{*}, \rho\right) \times B\left(t_{m}^{*}, \rho\right), 0\right) \\
& \neq 0
\end{aligned}
$$

Combining (6.7) with $(6.3)$ and $(6.4)$ we deduce that if $\varepsilon$ is small enough there exists $\left(\mathbf{s}^{\varepsilon}, \mathbf{t}^{\varepsilon}\right)$ such that $\nabla \mathcal{F}_{\varepsilon}\left(\mathbf{s}^{\varepsilon}, \mathbf{t}^{\varepsilon}\right)=0$. In particular, $\mathbf{s}^{\varepsilon}=\left(s_{1}^{\varepsilon}, \ldots, s_{m}^{\varepsilon}\right) \rightarrow$ $\left(\zeta_{1}^{*}, \ldots, \zeta_{m}^{*}\right)$ as $\varepsilon \rightarrow 0$. That concludes the proof.

Let us prove 6.2.

Using a smooth extension of the function $a(x)$ we can perform a Taylor expansion around every point $s_{i}$ to obtain that

$$
a\left(\zeta_{i}\right)=a\left(s_{i}\right)+|\ln (\varepsilon)|^{-1} t_{i} \partial_{\nu} a\left(s_{i}\right)+\mathcal{O}\left(\left[|\ln (\varepsilon)|^{-1} t_{i}\right]^{2}\right) .
$$

On the other hand, from Corollary 1 and the regularity in 2.7) of the function $R(z)$ described in $(2.6)$, we find that for any $\alpha \in(0,1)$ and any $\zeta_{i}$ as in 6.1 ,

$$
H\left(\zeta_{i}, \zeta_{i}\right)=4 \ln \left(\ln \left(\frac{1}{\varepsilon}\right)\right)+4 \ln \left(\frac{1}{2 t_{i}}\right)+\mathrm{z}\left(s_{i}\right)+\mathcal{O}\left(\left[|\ln (\varepsilon)|^{-1} t_{i}\right]^{\alpha}\right) .
$$

On the other hand, since $R \in C^{\infty}\left(\mathbb{R}^{2}-\{0\}\right)$, we can use expression 2.5), Proposition 1 and the fact that $\left|s_{i}-s_{j}\right| \geq c>0$, to obtain the expansion

$$
\begin{aligned}
G\left(\zeta_{i}, \zeta_{j}\right) & =-4 \ln \left(\left|s_{i}-s_{j}\right|\right)+\tilde{z}\left(s_{i}, s_{j}\right) \\
& +\frac{1}{\ln \left(\frac{1}{\varepsilon}\right)}\left[4 t_{j} R\left(s_{i}-s_{j}\right) \cdot\left(D \gamma\left(s_{j}\right) \cdot \nu\left(s_{j}\right)\right)+\nabla \tilde{z}\left(s_{i}, s_{j}\right) \cdot\left(t_{i} \nu\left(s_{i}\right), t_{j} \nu\left(s_{j}\right)\right)\right] \\
& +o\left(|\ln (\varepsilon)|^{-1}\left[\left|t_{i}\right|+\left|t_{j}\right|\right]\right) .
\end{aligned}
$$


Hence, from (5.5), putting together expressions 6.8, 6.9 and 6.10, we get

$$
\begin{aligned}
\mathcal{F}_{0}(\mathbf{s}, \mathbf{t}) & =\sum_{i=1}^{m}\left[\mathrm{c}_{0}+\mathrm{c}_{1} \ln \left(\ln \left(\frac{1}{\varepsilon}\right)\right)+\mathrm{c}_{2} \ln \left(\frac{1}{\varepsilon}\right)\right] a\left(s_{i}\right) \\
& +16 \pi \sum_{i=1}^{m}\left[\ln \left(t_{i}\right) a\left(s_{i}\right)+\left(1+\frac{\ln \left(t_{i}\right)}{\ln \left(\frac{1}{\varepsilon}\right)}\right) t_{i} \partial_{\nu} a\left(s_{i}\right)\right] \\
& -4 \pi \sum_{i=1}^{m}\left[\mathrm{z}\left(s_{i}\right)-\sum_{j \neq i} 4 \ln \left(\left|s_{i}-s_{j}\right|\right)+\tilde{z}\left(s_{i}, s_{j}\right)\right]\left(a\left(s_{i}\right)+\frac{t_{i}}{\ln \left(\frac{1}{\varepsilon}\right)} \partial_{\nu} a\left(s_{i}\right)\right) \\
& +\mathcal{O}\left(|\ln (\varepsilon)|^{-\alpha}|\mathbf{t}|^{\alpha}\right)
\end{aligned}
$$

for some constants $c_{0}, c_{1}$ and,$c_{2}>0$ and estimate $(6.2)$ follows.

This concludes the proof of the Theorem.

We next proceed with the proof of Theorem 2 .

Proof (Proof of Theorem 2.) By Proposition 4 we have to find a critical point $\boldsymbol{\zeta}^{\varepsilon}:=\left(\zeta_{1}^{\varepsilon}, \ldots, \zeta_{m}^{\varepsilon}\right) \in \partial \Omega \times \cdots \times \partial \Omega$ of the reduced energy $\mathcal{F}_{\varepsilon}$. By $(5.6)$ we have that $\mathcal{F}_{\varepsilon}$ reduces to

$$
\mathcal{F}_{\varepsilon}(\boldsymbol{\zeta})=|\ln \varepsilon|\left[8 \pi \sum_{i=1}^{m} a\left(\zeta_{i}\right)+o(1)\right]
$$

$C^{1}$-uniformly in $\left\{\left(\zeta_{1}, \ldots, \zeta_{m}\right) \in \partial \Omega \times \cdots \times \partial \Omega:\left|\zeta_{i}-\zeta_{j}\right| \geq c\right.$ for any $\left.i \neq j\right\}$. The claim follows from the fact that $a$ has $m$ different strict local maxima or local minima points $\zeta_{1}^{*}, \ldots, \zeta_{m}^{*}$ on the boundary $\partial \Omega$ which are stable under $C^{1}$-perturbation, using a degree argument as in the proof of Theorem 1 In particular, there exists a critical point $\left(\zeta_{1}^{\varepsilon}, \ldots, \zeta_{m}^{\varepsilon}\right)$ of $\mathcal{F}_{\varepsilon}$ such that each $\zeta_{i}^{\varepsilon} \rightarrow \zeta_{i}^{*}$ for $i=1, \ldots, m$.

We next proceed with the proof of Theorem 3

Proof (Proof of Theorem 3.) By Proposition 4 we have to find a critical point $\boldsymbol{\zeta}^{\varepsilon}:=\left(\zeta_{1}^{\varepsilon}, \ldots, \zeta_{m}^{\varepsilon}\right) \in \partial \Omega \times \cdots \times \partial \Omega$ of the reduced energy $\mathcal{F}_{\varepsilon}$. By $(5.6)$ we have that $\mathcal{F}_{\varepsilon}$ reduces to

$$
\mathcal{F}_{\varepsilon}(\boldsymbol{\zeta})=8 \pi|\ln \varepsilon| \sum_{i=1}^{m} a\left(\zeta_{i}\right)-2 \pi \sum_{\substack{j, i=1 \\ j \neq i}}^{m} a\left(\zeta_{i}\right) G\left(\zeta_{i}, \zeta_{j}\right)+o(1)
$$

$C^{0}$-uniformly in $\left\{\left(\zeta_{1}, \ldots, \zeta_{m}\right) \in \partial \Omega \times \cdots \times \partial \Omega:\left|\zeta_{i}-\zeta_{j}\right| \geq c|\ln \varepsilon|^{-\kappa}\right.$ for any $\left.i \neq j\right\}$.

Arguing exactly as in Lemma 7.1 of 33 we prove that $\mathcal{F}_{\varepsilon}$ has a local maximum point $\left(\zeta_{1}^{\varepsilon}, \ldots, \zeta_{m}^{\varepsilon}\right)$ such that each $\zeta_{i}^{\varepsilon} \rightarrow \zeta_{0}$ as $\varepsilon \rightarrow 0$. 


\section{Appendix}

The following technical lemma is rather standard, but for the sake of completeness, we include the details of the proof.

Lemma 3 For every $x, \zeta \in \partial \Omega$, define $g(x, \zeta)$ as

$$
g(x, \zeta):=\left\{\begin{array}{cc}
\frac{\nu(x) \cdot(x-\zeta)}{|x-\zeta|^{2}}, & x \in \partial \Omega-\{\zeta\}, \\
\frac{1}{2} k_{\partial \Omega}(\zeta), & x=\zeta,
\end{array}\right.
$$

where $k_{\partial \Omega}(y)$ is the signed curvature of $\partial \Omega$ at $y$. Then for every $\zeta \in \partial \Omega$, $g(\cdot, \zeta) \in C^{\infty}(\partial \Omega)$. Even more, the mapping $\zeta \in \partial \Omega \mapsto g(\cdot, \zeta)$ belongs to $C^{1}\left(\partial \Omega ; C^{1}(\partial \Omega)\right)$.

Proof Recall that $\partial \Omega$ is smooth. Let $\zeta \in \partial \Omega$ be arbitrary. After a translation and a rotation, if necessary, we may assume that $\zeta=(0,0) \in \mathbb{R}^{2}$ and that $\nu(\zeta)=(0,1)$. Hence, there exists $R>0, \delta>0$ small and a function $\mathrm{p}$ : $(-\delta, \delta) \rightarrow \mathbb{R}$ satisfying

$$
\mathrm{p} \in C^{\infty}(-\delta, \delta), \quad \mathrm{p}(0)=0, \quad \mathrm{p}^{\prime}(0)=0
$$

and such that

$$
\Omega \cap B_{R}(\zeta)=\left\{\left(x_{1}, x_{2}\right):-\delta<x_{1}<\delta, \quad \mathrm{p}\left(x_{1}\right)<x_{2}\right\} \cap B_{R}(0,0) .
$$

The inner unit normal vector in $\partial \Omega \cap B_{R}(\zeta)$ is computed as

$$
\nu\left(x_{1}, \mathrm{p}\left(x_{1}\right)\right)=\frac{\left(-\mathrm{p}^{\prime}\left(x_{1}\right), 1\right)}{\sqrt{1+\left|\mathrm{p}^{\prime}\left(x_{1}\right)\right|^{2}}}, \quad x_{1} \in(-\delta, \delta),
$$

so that for $x_{1} \in(-\delta, \delta)$

$$
g\left(x_{1}, \mathrm{p}\left(x_{1}\right), \zeta\right)=\frac{1}{\sqrt{1+\left|\mathrm{p}^{\prime}\left(x_{1}\right)\right|^{2}}} \cdot \frac{\mathrm{p}\left(x_{1}\right)-x_{1} \mathrm{p}^{\prime}\left(x_{1}\right)}{x_{1}^{2}+\mathrm{p}^{2}\left(x_{1}\right)} .
$$

Since $\mathrm{p}$ is smooth, we use Taylor's expansion together with the fact that $\mathrm{p}(0)=\mathrm{p}^{\prime}(0)=0$ to find that

$\mathrm{p}\left(x_{1}\right)=x_{1}^{2} \int_{0}^{1}(1-t) \mathrm{p}^{\prime \prime}\left(t x_{1}\right) d t=x_{1}^{2} \mathrm{r}\left(x_{1}\right), \quad \mathrm{p}^{\prime}\left(x_{1}\right)=x_{1} \int_{0}^{1} \mathrm{p}^{\prime \prime}\left(t x_{1}\right) d t=x_{1} \mathrm{q}\left(x_{1}\right)$,

where

$\mathrm{r}\left(x_{1}\right):=\int_{0}^{1}(1-t) \mathrm{p}^{\prime \prime}\left(t x_{1}\right) d t, \quad \mathrm{q}\left(x_{1}\right):=\int_{0}^{1} \mathrm{p}^{\prime \prime}\left(t x_{1}\right) d t, \quad$ for $\quad-\delta<x_{1}<\delta$.

Putting together (7.1) and (7.2) we obtain

$$
\frac{\mathrm{p}\left(x_{1}\right)-x_{1} \mathrm{p}^{\prime}\left(x_{1}\right)}{x_{1}^{2}+\mathrm{p}\left(x_{1}\right)^{2}}=\frac{\mathrm{r}\left(x_{1}\right)-\mathrm{q}\left(x_{1}\right)}{1+x_{1}^{2} \mathrm{r}^{2}\left(x_{1}\right)}
$$


which is a smooth function in $(-\delta, \delta)$.

By succesive differentiation of the identity in 7.3 , we conclude that $g(x, \zeta)$ is smooth and also notice that

$$
\lim _{x_{1} \rightarrow 0} g\left(\left(x_{1}, p\left(x_{1}\right)\right), \zeta\right)=-\frac{1}{2} \mathrm{p}^{\prime \prime}(0)=\frac{1}{2} k_{\partial \Omega}(0,0) .
$$

Finally from the smoothness of the tangent bundle of $\partial \Omega, T(\partial \Omega)$, we obtain that $\zeta \mapsto g(\cdot, \zeta)$ belongs to $C^{1}\left(\partial \Omega ; C^{1}(\partial \Omega)\right)$ and this concludes the proof of the lemma.

Acknowledgements The research of the first author was supported by the Grant 1300863S of the Grant Agency of the Czech Republic. The research of the second author was partially supported by GNAMPA.

\section{References}

1. Amann E. A note on degree theory for gradient mappings. Proceedings of the American Mathematical Society. Volume 85, Number 4, 591-595 1982.

2. Bellomo M., A. Bellouquid, Y. TaO And M. Winkler Toward a mathematical theory of Keller-Segel models of pattern formation in biological tissues Math. Models Methods Appl. Sci. 25, 1663-1763 (2015).

3. BILER P. Local and global solvability of some parabolic system modeling chemotaxis. Adv. Math. Sci. Appl. 8 715-43. 1998.

4. Brenner M. P., Constantin P., Kadanoff L. P., Schenkel A. and Venkataraman S. C. Diffusion, attraction and collapse. Nonlinearity 12 1071-98. 1999 .

5. Brezis, H. Functional analysis, Sobolev spaces and partial differential equations. Universitext. Springer, New York, 2011.

6. Childress S. Chemotactic Collapse in Two Dimensions (Lecture Notes in Biomathematics) vol 55 (Berlin: Springer) pp 61-8. 1984.

7. Childress S. And Percus J. K. Nonlinear aspects of chemotaxis Math. Biosci. 56 217-37. 1981.

8. Corrias L., Perthame B. And ZaAg H. Global solutions of some chemotaxis and angiogenesis systems in high space dimensions Milan J. Math. 72 1-28. 2004.

9. Del Pino M., Kowalczyk M. and Musso M. Singular limits in Liouville-type equations Calc. Var. Partial Diff. Eqns 24 45-82. 2005.

10. Del Pino M., Pistoia A. And Vaira G. Large mass boundary condensation patterns in the stationary Keller-Segel system arXiv 1403.2511

11. Del Pino M. And Wei, J. C. Collapsing steady states of the Keller-Segel system. Nonlinearity 19, no. 3, 661-684. 2006.

12. Di Nezza E., Palatucci G. and Valdinoci E. Hitchhiker's guide to the fractional Sobolev spaces. Bull. Sci. Math. 136, no. 5, 521-573. 2012.

13. Dolbeault J. and Perthame B. Optimal critical mass in the two-dimensional KellerSegel model in R2 C. R. Math. Acad. Sci. Paris 339 611-6. 2004

14. Esposito P., Grossi M. And Pistoia A. On the existence of blowing-up solutions for a mean field equation Ann. Inst. H. Poincaré Anal. Non Lineaire. 22 227-57. 2005.

15. Guerra I. And Peletier M. Self-similar blow-up for a diffusion-attraction problem Nonlinearity 17 2137-62. 2004.

16. Gilbarg D. And Trudinger N.S. Elliptic partial differential equations of second order. Reprint of the 1998 edition. Classics in Mathematics. Springer-Verlag, Berlin, 2001.

17. Henry D. Geometric Theory of Semilinear Parabolic Equations (Berlin: Springer) 1981.

18. Herrero M.A. AND VelazQuez J.J.L. Singularity patterns in a chemotaxis model Math. Ann. 306 583-623. 1996. 
19. Herrero M.A. And Velazquez J.J.L. Chemotactic collapse for the Keller-Segel model J. Math. Biol. 35 177-96. 1996.

20. Herrero M.A. and Velazquez J.J.L. A blow-up mechanism for a chemotaxis model Ann. Scuola Norm. Sup.Pisa IV 35 633-83. 1997.

21. Horstmann D. On the existence of radially symmetric blow-up solutions for the KellerSegel model J. Math. Biol. 44 463-78. 2002.

22. JAGER W. AND LuCKhaus S. On explosions of solutions to a system of partial differential equations modelling chemotaxis Trans. Am. Math. Soc. 329 819-24. 1992.

23. Keller E.F. AND SEgEL L.A. Initiation of slime mold aggregation viewed as an instability J. Theor. Biol. 26 399-415. 1970.

24. Molle R. and Pistoia A. Concentration phenomena in elliptic problems with critical and supercritical growth. Adv. Differential Equations 8 (2003), no. 5, 547-570.

25. Nagai T., Senba T. And Yoshida K. Application of the Trudinger-Moser inequality to a parabolic system of chemotaxis Funckcjal. Ekvac. 40 411-33. 1997.

26. Pistoia A. and Vaira G. Steady states with unbounded mass of the Keller-Segel system. Proc. Roy. Soc. Edinburgh Sect. A 145 (2015), no. 1, 203-222.

27. SchaAf R. Stationary solutions of chemotaxis systems. Trans. Am. Math. Soc. 292 531-56. 1985.

28. Senba T. AND Suzuki T. Some structures of the solution set for a stationary system of chemotaxis Adv. Math. Sci. Appl. 10 191-224. 2000.

29. Tеmam R. Infinite-Dimensional Dynamical Systems in Mechanics and Physics (New York: Springer). 1988.

30. WANG G. AND WEI J.C. Steady state solutions of a reaction-diffusion system modeling Chemotaxis Math. Nachr. 233-234 221-36. 2002.

31. WANG Y. AND WEI L. Multiple boundary bubbling phenomenon of solutions to a Neumann problem. Adv. Differential Equations 13, no. 9-10, 829-856. 2008.

32. Wei J.C., Ye D. AND ZhOU F. Analysis of boundary bubbling solutions for an anisotropic Emden-Fowler equation. Ann. Inst. H. Poincaré Anal. Non Linéaire 25, no. 3, 425-447, 2008.

33. Wei J.C., Ye D. AND Zhou F. Bubbling solutions for an anisotropic Emden-Fowler equation. Calc. Var. Partial Differential Equations 28, no. 2, 217-247. 2007. 